\title{
Absence of a Dissipative Quantum Phase Transition in Josephson Junctions
}

\author{
A. Murani, ${ }^{1}$ N. Bourlet, ${ }^{1}$ H. le Sueur, ${ }_{3}^{1}$ F. Portier, ${ }^{1}$ C. Altimiras $\odot,{ }^{1}$ D. Esteve, ${ }^{1}$ H. Grabert, ${ }^{2}$ \\ J. Stockburger ${ }^{3}{ }^{3}$ J. Ankerhold, ${ }^{3}$ and P. Joyez $\odot^{1, *}$ \\ ${ }^{1}$ Université Paris-Saclay, CEA, CNRS, SPEC, 91191 Gif-sur-Yvette Cedex, France \\ ${ }^{2}$ Physikalisches Institut, Universität Freiburg, Hermann-Herder-Straße 3, 79104 Freiburg, Germany \\ ${ }^{3}$ Institute for Complex Quantum Systems and IQST, University of Ulm, 89069 Ulm, Germany
}

(Received 7 May 2019; accepted 21 February 2020; published 3 April 2020)

\begin{abstract}
Half a century after its discovery, the Josephson junction has become the most important nonlinear quantum electronic component at our disposal. It has helped reshape the International System of Units around quantum effects and is used in scores of quantum devices. By itself, the use of Josephson junctions in volt metrology seems to imply an exquisite understanding of the component in every aspect. Yet, surprisingly, there have been long-standing subtle issues regarding the modeling of the interaction of a junction with its electromagnetic environment. Here, we find that a Josephson junction connected to a resistor does not become insulating beyond a given value of the resistance due to a dissipative quantum phase transition, as is commonly believed. Our work clarifies how this key quantum component behaves in the presence of a dissipative environment and provides a comprehensive and consistent picture, notably regarding the treatment of its phase.
\end{abstract}

DOI: 10.1103/PhysRevX.10.021003

Subject Areas: Superconductivity

\section{INTRODUCTION}

In 1983, Schmid [1] predicted that a dissipation-driven quantum phase transition (DQPT) should occur for any Josephson junction (JJ) connected to a resistance $R$ : When $R>R_{Q}=h / 4 e^{2} \simeq 6.5 \mathrm{k} \Omega$, the junction should be insulating at zero temperature, while if $R<R_{Q}$, the junction should be superconducting (see Fig. 1). The prediction was made more precise shortly after by Bulgadaev [2], and since then, many theoretical works using different techniques [3-12] have further confirmed it. Attempts to investigate this prediction experimentally are scarce [13-15], and these early experiments were all affected by technical limitations (see the Appendix A) that made their interpretation debatable. In this work, we revisit this prediction using well-controlled linear response measurements on the insulating side of the phase diagram, and we find no sign of the junctions becoming insulating. By revisiting the theory, we provide arguments explaining why, actually, no superconducting-to-insulating transition is expected, and we propose an alternative comprehensive physical picture for this system.

Let us first motivate our work by explaining why the predicted phase diagram is problematic. The left axis in the

*philippe.joyez@cea.fr

Published by the American Physical Society under the terms of the Creative Commons Attribution 4.0 International license. Further distribution of this work must maintain attribution to the author(s) and the published article's title, journal citation, and DOI.
Schmid-Bulgadaev (SB) phase diagram [Fig. 1(b)] corresponds to $R \rightarrow \infty$, where we can simply remove the resistor from the circuit. In this limit, we are left with only the junction represented as a pure Josephson element in parallel with the junction's geometric capacitor $C$ defining the charging energy $E_{C}=(2 e)^{2} / 2 C$. Such a disconnected junction is known as a Cooper pair box (CPB) in the domain of quantum circuits; it behaves as a nonlinear oscillator and has been extensively investigated theoretically and experimentally [16-19]. In particular, for any junction with a nonzero Josephson coupling $E_{J}$, a CPB has finite charge fluctuations through the junction, in contradiction with it being on the insulating side of the phase transition, and it was shown that one can indeed drive finite ac supercurrents through the junction [20]. Furthermore, since the anharmonicity of the CPB vanishes upon increasing the ratio $E_{J} / E_{C}$ [18], one expects (at least in the large $E_{J} / E_{C}$ range) the effect of a finite parallel resistance $R$ on this nonharmonic oscillator to be similar to that on a harmonic oscillator $[21,22]$ : When $R$ is varied, the phase and charge fluctuations have no abrupt change at $R=R_{Q}$. Approaches that go beyond considering the junction as a pure inductor $[23,24]$ confirm this intuition down to the moderately large $E_{J} / E_{C}$ range: They predict a superconductive junction that smoothly retrieves the "bare" (with no resistor) CPB behavior as the environment impedance gets large and cold. More generally, any Josephson junction connected to a large impedance $Z$ is intuitively expected to smoothly recover the (superconducting) behavior of the CPB in the $Z \rightarrow \infty$ limit. This was 

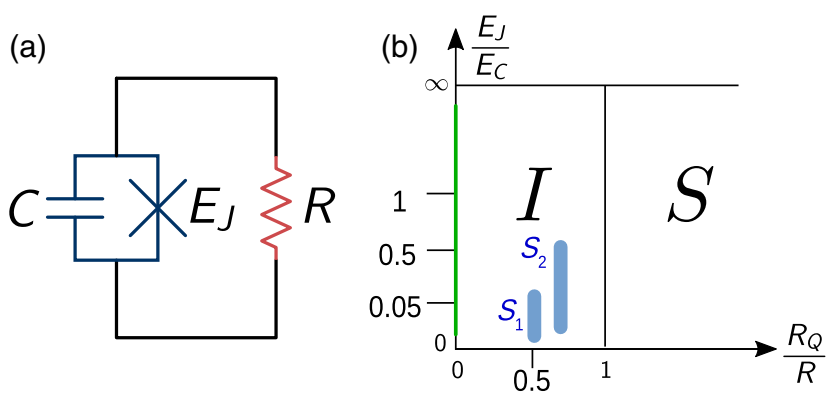

FIG. 1. (a) A Josephson junction connected to a resistor $R$ (abbreviated as $\mathrm{JJ}+R$ ). The junction's capacitance $C$ determines the charging energy $E_{C}=(2 e)^{2} / 2 C$, while the transparency of the tunnel barrier and the superconducting gap set its Josephson coupling energy $E_{J}$. (b) Sketch of the SchmidBulgadaev phase diagram for the circuit in (a). In the phase $I(S)$, the junction is predicted to be insulating (superconducting) at zero temperature. The insulating phase is paradoxical because the left axis (green line, where $R=\infty$ ) is the location of the Cooper pair box family of superconducting qubits for which it is well known that the junction is superconducting. Similarly, our samples $S_{1}$ and $S_{2}$ are found to remain superconducting when lowering the temperature, even though they are supposed to be well inside the insulating phase.

confirmed theoretically in the specific case of a purely inductive environment in Ref. [25]. In summary, several known theoretical results [18,21-25], many experimental results [16-18,20], and intuitive expectations in simple limits are consistent among themselves and conflict with the prediction of the insulating phase shown in Fig. 1(b).

\section{EXPERIMENT}

In order to test the SB prediction, we designed an experiment that closely implements the circuit of Fig. 1(a) while allowing us to probe the linear response of Josephson junctions in ac. A schematics of the experiment and a micrograph of a sample are shown in Fig. 2, and the main sample parameters are given in Table I. Instead of a single junction, we use a superconducting quantum interference device (SQUID) behaving as an effective tunable Josephson junction: By applying a magnetic flux $\Phi$ in the SQUID loop, its Josephson coupling energy is tuned as $E_{J} \simeq E_{J \max }\left|\cos \left(\pi \Phi / \Phi_{0}\right)\right|$ with $\Phi_{0}=h / 2 e$ the flux quantum. The input capacitor $C_{c}$ is chosen small enough that, at the measurement frequency, it essentially converts the input ac signal into a current source for the parallel junction-capacitance-resistance system. This current is split between these components according to their admittance. The fraction of the current flowing through the resistor is routed off chip to a microwave bias tee. The dc port of the bias tee is shorted to ground, closing the circuit in $\mathrm{dc}$ and ensuring there is no dc bias applied on the junction. At the high-frequency port of the bias tee, the ac signal coming from the resistor is sent through circulators

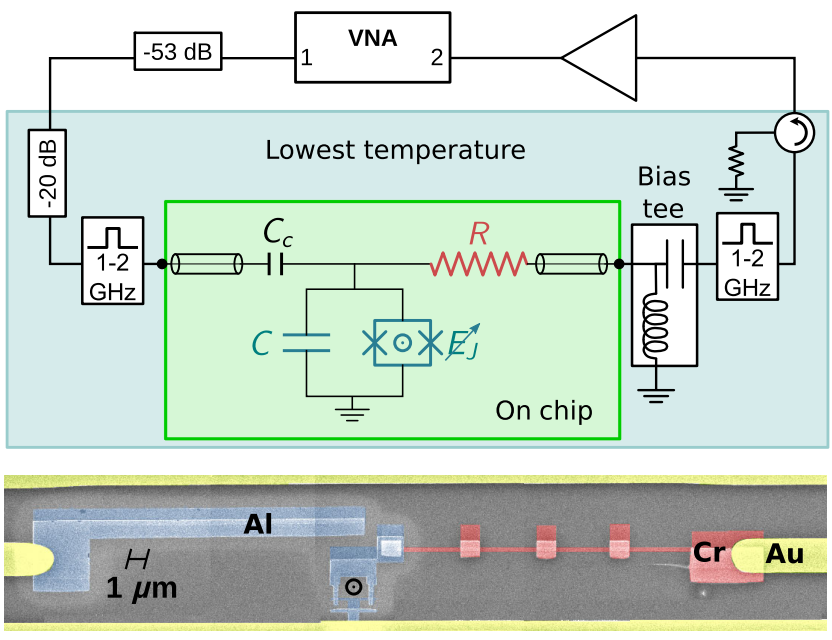

FIG. 2. Top: Simplified schematics of the experimental setup. Bottom: One of the samples measured. Two SEM micrographs are stitched to show the entire central part and colorized to evidence the different metals used (see Appendix B for fabrication details).

and filters to a chain of microwave amplifiers with an overall gain of $106 \mathrm{~dB}$. We used microwave simulations of the circuit to check that in this design, the actual impedance seen by the junction is close to $R \| C$ up to frequencies well above $(R C)^{-1}$ (note that the impedance to ground of the circuit following the resistor is negligible compared to $R$ at all frequencies). We used a vector network analyzer to perform continuous-wave homodyne measurements of the transmission $S_{21}$ through the sample. Although in this setup we measure variations of the fraction of the ac current flowing through the resistor, they are directly related to the variations of the junction admittance (see Appendix E).

The operating conditions of the experiment are subject to constraints that we now detail. First, in order to improve our sensitivity to the junction's admittance [26], the measurements need to be performed at a frequency well below the "plasma frequency" $\omega_{p}=\left(C L_{J}^{\text {eff }}\right)^{-1 / 2}$ of the junction, so that, as seen from the input capacitance, the ac current through $C$ is negligible. The current is then essentially divided between the resistor and the junction's effective inductance $L_{J}^{\text {eff }}$, should it exist, in proportion of their respective admittance $1 / R$ and $1 / i L_{J}^{\text {eff }} \omega$. We selected an operating frequency of order $1 \mathrm{GHz}$ in order to simultaneously fulfill this constraint (except in the vicinity of the maximal frustration of the SQUID) and have a reasonably

TABLE I. Main sample parameters. See Appendix B for details on their determination.

\begin{tabular}{lcccc}
\hline \hline Sample & $E_{c} / k_{B}(\mathrm{~K})$ & $E_{J \max } / k_{B}(\mathrm{~K})$ & $R(\mathrm{k} \Omega)$ & $C_{c}(\mathrm{fF})$ \\
\hline 1 & 2.6 & 0.12 & 12 & 0.3 \\
2 & 0.64 & 0.39 & 8 & 0.3 \\
\hline \hline
\end{tabular}


good noise temperature for our microwave amplifier. Second, since we aim to probe the linear response of the junction at equilibrium, the ac phase excursion must be $\delta \varphi \ll 2 \pi$, so that the junction is properly described by an admittance $1 / i L_{J}^{\text {eff }} \omega$. Assuming the worst case where all the current flows through the resistor, this inequality restricts the ac amplitude at the sample input $V_{\text {in }} \ll \Phi_{0} / R C_{c}$ (that is, $P_{\text {in }} \ll-50 \mathrm{dBm}$ for the values used in the experiment; see below). Correspondingly, all the measurements that we show here are taken in the low-power limit where $S_{21}$ no longer depends on the input power (see Appendix D). The last constraint also restricts the admissible input power: The Joule power dissipated by ac current flowing through the resistor should not raise its temperature significantly. We use the results of Ref. [27] to estimate the electronic heating. Neglecting electron-phonon cooling in the resistor, for the maximum $S_{21}$ value of $-50 \mathrm{~dB}$, and at the input power of $-70 \mathrm{dBm}$ used for the sample 2 data at the lowest temperature $\left(T_{\mathrm{ph}}=13 \mathrm{mK}\right)$ in Fig. 3, one predicts an upper bound for the electronic temperature rise of approximately $1.0 \mathrm{mK}$ (0.5 $\mathrm{mK}$ for sample 1) close to the junction (see Appendix C). Note that for such a low power level, the signal-to-noise ratio at the input of the first cryogenic HEMT amplifier is such that each data point necessitates averaging for about $20 \mathrm{~min}$. Above about $50 \mathrm{mK}$, electron-phonon cooling becomes effective (see Appendix C); it is then possible to speed up the measurement by increasing the excitation amplitude (still remaining in the linear regime) without raising the electronic temperature.

In Fig. 3, we show the transmission $S_{21}$ for the two samples we measured, for different flux through the

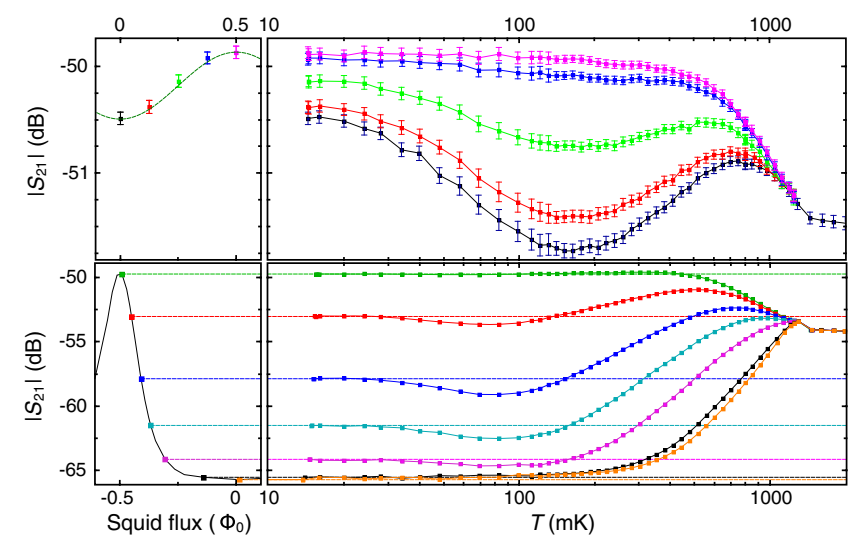

FIG. 3. Measured modulus of the transmission $S_{21}$ (as a power ratio) for sample 1 (top panels) and 2 (bottom panels). Left panels: $\left|S_{21}\right|$ as a function of the flux through the SQUIDs at the base temperature. The modulation is periodic with the flux (data not shown), as usual for a SQUID; only half a period is represented. Note that the position of the zero flux is different in the top and bottom panels. Right panels: $\left|S_{21}\right|$ for several flux values (using the same colors as on the left panels) as a function of the temperature. For sample 2, the error bars are smaller than the symbols used (note the larger vertical scale).
SQUID and different temperatures. On the left panels, we show $S_{21}$ as a function of the flux in the SQUIDs at the lowest temperature. We observe that when the flux is zero in the SQUID, the junction has the highest admittance ( $S_{21}$ minimum), whereas its admittance is minimum when the SQUID is frustrated with half a flux quantum in the loop. On the right panels, we show the temperature dependence of $S_{21}$ for several values of the flux in the SQUIDs. We observe that in the low temperature range, for any fixed value of the flux, $S_{21}$ reaches plateaus indicating that the junction admittance saturates to a finite value. In other words, at low temperature, the modulation of $S_{21}$ with the flux proves that the SQUID still carries supercurrent, and it shows no tendency to become insulating at lower temperatures.

\section{DISCUSSION}

Would the predicted insulating phase exist, the junctions would be in the quantum critical regime where one expects the junction admittance to follow a power law of the temperature [28]. This is clearly not the case in our experiments. In a totally independent experiment with a different objective, Grimm et al. [29] have recently observed that a SQUID with $E_{J}^{\max } / E_{C} \simeq 0.3$ in series with a $32-\mathrm{k} \Omega$ resistance $\left(R_{Q} / R \simeq 0.2\right)$ had a clear dc supercurrent branch that was modulated with the flux. We consider their observation to support our results.

Together with the known $R \rightarrow \infty$ limit of qubits and the observed superconducting junctions at $E_{J} / E_{C} \gtrsim 7$ and $R_{Q} / R \sim 0.6$ in Refs. [14,30] (see Appendix A), we conclude that the experimental observations are consistent with a complete absence of the predicted insulating phase.

We now turn to theoretical considerations. In the first step, we revisit the framework in which the SB prediction of a superconducting-to-insulating phase transition was made. In the second step, we explain the exact nature of the predicted transition and provide arguments according to which JJs are actually not expected to become insulating in any Ohmic environment.

The SB prediction was cast using the model introduced by Caldeira and Leggett (CL) [31], which describes a Josephson junction and its capacitor (forming a CPB) analogous to a massive particle in a washboard potential, coupling the particle position (the junction phase) to a bath of harmonic oscillators that provide viscous damping. The corresponding Hamiltonian is

$$
H=E_{C} N^{2}-E_{J} \cos \varphi+\sum_{n} 4 e^{2} \frac{N_{n}^{2}}{2 C_{n}}+\frac{\hbar^{2}}{4 e^{2}} \frac{\left(\varphi_{n}-\varphi\right)^{2}}{2 L_{n}},
$$

where $\varphi$ (resp. $N$ ) denotes the junction's phase (resp. number of Cooper pairs on the junction capacitance) which are conjugate $[\varphi, N]=i$, and the $\varphi_{n}$ (resp. $N_{n}$ ) denote the phase (resp. dimensionless charge) of the harmonic 
oscillators. $H$ is not invariant upon $\varphi \rightarrow \varphi+2 \pi$, so that values of $\varphi$ differing by $2 \pi$ are naturally regarded as distinguishable states of the junction, and $\varphi$ is said to be an "extended phase." Correspondingly, $N$ has its spectrum in $\mathbb{R}$, and we call it an extended charge too.

A unitary transformation $H^{\prime}=U^{\dagger} H U$ with $U=$ $\exp \left(i \varphi N_{R}\right)$ and $N_{R}=\sum_{n} N_{n}$ (the charge passed through the resistor) yields another Hamiltonian of interest

$$
H^{\prime}=E_{C}\left(N-N_{R}\right)^{2}-E_{J} \cos \varphi+\sum_{n} 4 e^{2} \frac{N_{n}^{2}}{2 C_{n}}+\frac{\hbar^{2}}{4 e^{2}} \frac{\varphi_{n}^{2}}{2 L_{n}},
$$

where the $\mathrm{CPB}$ now couples to the environment through $N$, here representing the number of transmitted Cooper pairs through the junction. Unlike $H, H^{\prime}$ is evidently invariant upon the discrete translation $\varphi \rightarrow \varphi+2 \pi$ so that the values of $\varphi$ differing by $2 \pi$ can be regarded as indistinguishable (wave functions in $\varphi$ are $2 \pi$ periodic), and the usual terminology is that $\varphi$ is a "compact phase." In principle, $\varphi$ can still be described as an extended variable, in which case the periodicity of the potential implies that wave functions in $\varphi$ are Bloch functions $\Psi_{q}(\varphi)=\sum_{n} a_{n}(q) e^{i(n+q) \varphi}$. Here, the "quasicharge" $q$ is a conserved quantity fixed by initial conditions. However, a Bloch function with quasicharge $q$ can be transformed to any other Bloch function by a global shift of the bath charge, and the resistance is translationally invariant in both charge and phase (this invariance being respected in the CL model [31]). Thus, in this resistively shunted JJ, states with different quasicharges can be considered degenerate in the sense that no measurement on the circuit can distinguish them after the initial charge shift of the bath has decayed [32]. Thus, one can choose to use only compact phase states $(q=0 \bmod 2 \pi)$ for convenience. In this case, $\mathrm{N}$ has a discrete spectrum in $\mathbb{Z}$ (even though there is no island in the circuit), and the Josephson coupling term can be written as $E_{J} \cos \varphi=\frac{1}{2} E_{J}\left(\sum_{N \in \mathbb{Z}}|N\rangle\langle N+1|+\right.$ H.c. $)$ as customary for CPBs, which we expect to recover in the $R \rightarrow \infty$ limit.

With these provisos, $H$ and $H^{\prime}$ operate on wave functions with different symmetries; they almost seem to describe different physical systems. This issue was known from the start, and several theory papers considered the suitability of either phase description for the system considered here, but no clear-cut answer emerged (for an overview, see Ref. [33]). However, a unitary transformation cannot break a symmetry of the system, and the contradiction resolves when one properly transforms the boundary and initial conditions together with the Hamiltonian [33,34]. Provided this transformation is carried out properly and barring any spontaneous symmetry breaking, $H$ and $H^{\prime}$ can be used indifferently to describe the system, and any valid state of the system should thus be representable with either $H$ or $H^{\prime}$. As we mention above, in the $R=\infty$ limit of the bare CPB, the phase is known to be compact; hence, by continuity, compact states are also the states to consider at finite $R$, unless one shows a spontaneous symmetry breaking of the discrete phase translation invariance occurs, a phenomenon also known as the "decompactification" $[6,35]$ of the phase (and which goes along with an "undiscretization" of the charge).

The SB theory is precisely all about dissipation causing spontaneous symmetry breaking; we now describe the core ideas of this theory. Close to the bottom axis of the phase diagram, in the so-called scaling limit where $E_{C} \rightarrow \infty$ (which constrains $N=N_{R}$ ), $H^{\prime}$ becomes equivalent to the tight-binding model used in Refs [3,4] (see Appendix G). In this model, at low friction (low $R$ ), the junction's zerotemperature reduced density matrix $\rho$ is completely delocalized in the discrete charge basis, and thus corresponds to a perfectly localized compact phase. For such a state, using an extended description for both charge and phase, the diagonal of $\rho$ is a Dirac comb in both charge and phase representation [Fig. 4(b), bottom right]. For $R>R_{Q}$, however, the discrete translational invariance symmetry of the charge is broken, and the charge localizes at a given value of $\langle N\rangle=\operatorname{Tr} \rho N$. In $\rho$, the result of this charge localization can be seen as multiplying the charge Dirac comb by a bell-shaped function $b$ and broadening each peak of the phase Dirac comb by convolving it with the Fourier transform of $b$ [Fig. 4(b), bottom left]. Across the transition, the charge fluctuations (the width of $b$ ) vary continuously [4], but the dc charge mobility $\mu$ (related to the charge fluctuations according to the standard Green-Kubo relations; see Appendix F) is predicted to
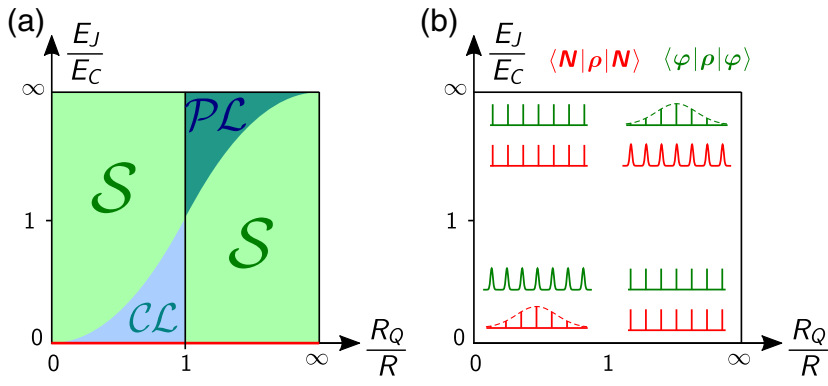

FIG. 4. (a) Reinterpreted Schmid-Bulgadaev phase diagram, in which the junction is superconducting everywhere, except for $E_{J}=0$. In the $\mathcal{S}$ parts, the junction is superconducting with a fully delocalized charge and, correspondingly, a fluctuationless (classical) compact phase. Partial charge (phase) localization occurs in the $\mathcal{C} \mathcal{L}(\mathcal{P} \mathcal{L})$ part. The classical phases $\mathcal{S}$ are artifacts which disappear when improving the model (see text and Appendix G). (b) Final description of the junction's behavior in the parameter space. Drawings are sketches of the diagonal elements of the junction's reduced density matrix in an extended description (red, charge representation; green, phase representation). Close to the left (right) half of the upper (lower) axis, they nearly take the form of Dirac combs where the phase is almost a classical variable. In the lower left (upper right) sector, partial charge (phase) localization occurs, as in (a). The density matrix evolves continuously, interpolating between these limits, without any phase transition. 
vanish (resp. diverge) for $R>R_{Q}$ (resp. $R<R_{Q}$ ) at $T=0$, hence, the prediction of a superconducting-to-insulating transition.

At the time of the prediction, this mobility argument was often associated with the simple picture of infinite polaronic trapping in the insulating phase $R>R_{Q}$ and the corresponding suppression of the coherence $E_{J}\langle\cos \varphi\rangle$ between charge states. More elaborate renormalization group (RG) flow arguments $[1-3,6,12,36]$ led to the conclusion that an immobilization of the junction charge indeed occurred in the whole domain where the cutoff frequency of the Ohmic damping is the fastest dynamics in the system, i.e., $E_{J} / E_{C}<$ $\left(R_{Q} / R\right)^{2}$ [see part $\mathcal{C} \mathcal{L}$ in Fig. 4(a); note that our experimental parameters are in this zone].

However, works on the closely related spin-boson problem (SBP; the CL model is an infinite-spin generalization of the SBP) have shown that the picture of infinite polaronic trapping is too naive. In this system, the spin and the bath entangle in the ground state, involving an infinite number of bosonic excitations and yielding resilient finite coherences (possibly very small) [37-40] that depend algebraically on the UV cutoff of the Ohmic bath. In the CL model itself, perturbation theory in $E_{J}$ shows as well that, while $E_{J}\langle\cos \varphi\rangle=0$ at zeroth order, $E_{J}\langle\cos \varphi\rangle=$ $\mathcal{O}\left(E_{J}^{2} / E_{C}\right)$ (as in the bare CPB) at the next order [41] for any $R>R_{Q} / 2$. Hence, it is no longer believed that the coherences vanish in the "insulating phase," and this has dramatic consequences: (i) It enables a finite supercurrent flow (as evidenced by our experiments), and (ii) previously calculated dc charge mobility does not describe the actual transport properties, because it does not take into account the inductive behavior associated with the supercurrent (see Appendix F). The qualitative explanation for the robustness of the coherence is that the inductive response of the junction shunts the low-frequency modes of the environment that were supposed to fully suppress the coherence [23]. In this new understanding of the (previously believed) insulating phase, the partially localized charge states are similar to those of the bare CPB, and they very naturally coincide with them in the $R \rightarrow \infty$ limit. The difference between the resistively shunted junction and the CPB with an island is that in the first case there is a degenerate continuum of localized charge states at all values of $\langle N\rangle$, while in the second case where no dc current can flow, $\langle N\rangle$ is pinned, and the ground state is unique.

Close to the top axis of the phase diagram, one follows similar reasoning in the "dual" picture [42], where charge and phase are interchanged. One then starts from a tightbinding description of Wannier states for the phase located in the different wells of the cosine potential (and where the strength of the friction is inverted [42]). Mirroring what occurs on the bottom axis, this duality predicts that the diagonal of $\rho$ is again a Dirac comb in both charge and phase representations [upper left of Fig. 4(b)] at low friction (large $R$ ) and that a smooth spontaneous symmetry breaking transition to partial "phase localization" occurs for $R<R_{Q}$ [part $\mathcal{P} \mathcal{L}$ in Fig. 4(a)]. We thus identify this transition as a progressive decompactification of $\varphi$. This shows that a generic decompactified phase state is the dual of a CPB state, i.e., a superposition of classical phase states differing by $2 \pi$ in several adjacent wells of the cosine. To our knowledge, this is the first time the decompactification process is clarified, and it is a key result as it shows this spontaneous symmetry breaking does not yield generic extended phase states, contrary to what was generally assumed so far (see Appendix H). In particular, Schmid and subsequent authors treated $\varphi$ as extended, which led them to attribute an insulating character to the "delocalized phase" in all the wells of the cosine (for $R>R_{Q}$ ). However, when considering a compact phase, the junction is insulating only when the phase is completely delocalized within one period (all coherences vanishing: $\langle\cos n \varphi\rangle=0$, $\forall n \in \mathbb{N}^{*}$ ), meaning that the diagonal of $\rho$ is completely flat in the phase representation.

In Fig. 4(a), we show our reinterpretation of the SB phase diagram, where the junction is superconducting everywhere, except at $E_{J} / E_{C}=0$, in the so-called scaling limit. Note that in actual implementations, $E_{C}$ is always finite, so that the insulating state of the scaling limit can be achieved only by choosing $E_{J}=0$, i.e., trivially, an already fully insulating junction (even in the normal state). This reinterpreted diagram is in agreement with experiments and resolves the conflicts mentioned in the Introduction. At this point, the vertical boundaries at $R=R_{Q}$ which remain from the SB prediction are continuous transitions from fluctuationless phase states to states having finite zero-point phase fluctuations, i.e., classical-to-quantum transitions. However, one can show these transitions arise from properties of the uncoupled bosonic bath (Ref. [3] and Appendix G), and one expects that a better treatment (taking into account the aforementioned entanglement of the junction with the bath) should restore finite phase fluctuations in the phases $S$, turning this transition into a crossover.

The emergent understanding of this system is represented pictorially in Fig. 4(b): The junction is superconducting everywhere, and its reduced density matrix evolves continuously as a function of the parameters, interpolating between the limit cases depicted. From this diagram, one sees that when the effective Josephson Hamiltonian is deemed adequate to model a Josephson junction (see Appendix I), the junction phase can be essentially regarded as compact (and one can use the discrete charge basis of a $\mathrm{CPB}$ ) below the main antidiagonal, while one expects a partial decompactification of the phase above that antidiagonal.

Obviously, generic extended phase states do not have the appropriate symmetries within this understanding. Consequently, assuming an extended phase to describe the low-energy states in such a system is at best approximate or it appeals to (perhaps unspoken) ingredients external to the CL model. Yet, many predictions (besides 
the DQPT) were made assuming an extended phase and have been checked to well describe the Josephson physics. This raises the question of when can one safely use such a description? A nonoperative answer is that such a description is fine as long as interference effects that would appear in a proper treatment of the phase (more or less complete) translation invariance play no significant role.

Before closing this discussion, let us comment on the striking dips observed in the temperature dependence of the transmitted power near $T \sim 100 \mathrm{mK}$ corresponding to a maximum of the junction admittance. They can be understood at a qualitative level using the usual charge description of the CPB (consistent with the above discussion), assuming the resistance is large enough. In the regime $E_{J} \ll E_{C}$ and at very low temperature, the state of the CPB is nearly a classical state at the minimum of a charging energy parabola with a given $N$. This state nevertheless has quantum fluctuations that can be computed by second-order perturbation theory, with virtual transitions through the neighboring charge states. This process results in an effective Josephson coupling for the ground state $E_{J}^{\mathrm{eff}}=E_{J}\langle\cos \varphi\rangle \propto E_{J}^{2} / E_{C}$, the energy denominator $E_{C}$ being the energy of the virtual states. At finite temperatures $k_{B} T \lesssim E_{C}$, low-energy modes of the resistance are thermally populated; they can lend their energy to the virtual state, lowering the energy denominator and thus increasing the effective Josephson coupling. At higher temperatures, thermal fluctuations eventually reduce the gap of $\mathrm{Al}$, reducing the Josephson coupling.

\section{METADISCUSSION}

Given that the present work contradicts more than 35 years of literature on the understanding of a Josephson junction in a resistive environment, one may rightfully wonder if alternative explanations of our results could exist. One can hypothesize that

(i) we might over- or misinterpret our experimental data and that of Grimm et al. [29] when we conclude that the junctions remain superconducting at low temperatures, and

(ii) there could be hidden flaws in our theoretical analysis of the CL model, which leads us to conclude that no insulating phase is expected in $\mathrm{JJ}+R$ systems,

such that the original SB prediction regarding JJs could stand. Within these hypotheses, signatures of the insulating state could, for instance, appear only out of the experimental windows for some reason to be worked out, making our experimental data compatible with the original prediction. However, we stress that the inconsistency of the insulating phase with the intuitive limits that we point to in the Introduction would still need to be addressed.

We thus encourage experimental and theoretical work in this domain that could complete, clarify, or correct our findings, in the hope that the community soon reaches consensus on the expected behavior of this key quantum component in the presence of an environment.

\section{CONCLUSIONS}

Our experimental results show no evidence of the superconducting-to-insulating DQPT in Josephson junctions predicted by Schmid and Bulgadaev, contrary to present widespread expectations. We provide theoretical arguments according to which the superconducting coherence in JJs is actually resilient to dissipation, thereby barring the occurrence of that DQPT in $\mathrm{JJ}+R$ systems (the DQPT does occur in nonsuperconducting 1D systems, however; see Appendix J). We reach a global and consistent qualitative description of JJs with an environmental impedance that dovetails all well-known limits. As an important by-product, our analysis for the first time clearly exposes how phase decompactification occurs in Josephson junctions. This shows that generic extended phase states are not rigorous solutions for this system, hopefully settling decades of controversies. Our work also highlights that there are presently no comprehensive and quantitative predictions for the effect of dissipation on the CPB able to reproduce our results. Finally, our results prompt for a critical reexamination of the works where the SchmidBulgadaev prediction regarding Josephson junctions was used to draw predictions for other systems such as superconducting nanowires proposed to implement quantum phase slip junctions [43-45].

\section{ACKNOWLEDGMENTS}

The authors are grateful to M. Devoret, B. Douçot, S. Florens, M. Hofheinz, C. Mora, I. Safi, H. Saleur, P. Simon, and N. Roch for stimulating discussions and suggestions. The technical assistance of Pascal Sénat and SPEC's Nanofabrication lab is acknowledged, and we thank other members of the SPEC's Quantronics group for their constant support. This work is supported in part by ANR Grant No. ANR-15-CE30-0021-01 and ANR-18CE47-0014-01, the ANR-DFG Grant JosephSCharli, and by the LabEx PALM Project No. ANR-10-LABX-0039PALM. J. A. and J. S. are supported by the German Science Foundation under Grant No. AN336/11-1 and the Center for Integrated Quantum Science and Technology (IQST). C. A. acknowledges funding from the European Research Council under the European Union's Horizon 2020 program (ERC Grant Agreement No. 639039).

\section{APPENDIX A: FORMER EXPERIMENTAL TESTS}

The SB prediction has been researched experimentally [13-15,30], but the scaling laws expected to be the hallmark of the predicted quantum critical regime have not been thoroughly investigated.

In these experiments, the junction and its Ohmic shunt resistance $R$ were typically "current biased" using a voltage 
source in series with a large resistor $R_{\text {bias }}>R$ and measured using a lock-in technique at frequencies $f_{\mathrm{LI}} \sim 100 \mathrm{~Hz}$ or below. Could such a setup properly measure the linear response of the junction?

For junctions with small critical current, it is well known that spurious noise in the setup rapidly reduces the apparent maximum supercurrent [46-48], and particularly so for underdamped junctions, i.e., when $E_{J} / E_{C} \gg\left(R_{Q} / R\right)^{2}$. However, even when the technical noises are completely eliminated, a lock-in measurement has intrinsic limitations when the junction's admittance becomes smaller than $1 / R$. In that case, keeping a small phase excursion in these setups requires an ac voltage excitation at the junction $V_{\text {ac }} \ll$ $\Phi_{0} f_{\mathrm{LI}}<1 \mathrm{pV}$ which, even taking into account the resistive bridge division $R /\left(R+R_{\text {bias }}\right)$, is several orders of magnitude smaller than required to have a sufficient signal-tonoise ratio in lock-in measurements. Thus, the former experiments aiming to test the DQPT could not properly measure the linear response of junctions with very low admittances: Several periods of the cosine were explored, rapidly averaging any small supercurrent to zero. In contrast, in our setup, measuring at much higher frequencies enables us to use larger excitation voltages while remaining in the linear phase response regime, even when the admittance of the junction becomes very low.

On the other hand, it is easy to observe the supercurrent branch of junctions having a large critical current, even with an imperfect setup, because the junction very effectively shunts noise. Indeed, the authors of Refs. [14,30] found that a superconducting branch was observed for all junctions supposed to be in the insulating phase, provided that $E_{J} / E_{C} \gtrsim 7$. At the time of this result, the discrepancy with the DQPT prediction was resolved by arguing that the observed superconducting state was a transient and that the true equilibrium insulating state would be reached only after a possibly cosmologically long time $[6,14,30]$. The argument given was that when the junction's (extended) phase starts localized in one well of the cosine potential, it will eventually delocalize in all other wells of the cosine by tunneling (and this delocalized state was assumed insulating), but the tunneling rate becomes immeasurably small for large $E_{J} / E_{C}$. However, when timescales become very long and energies very small, one should seriously reconsider all other approximations made in the modeling, such as, for instance, neglecting the level separation in the electrodes. When considering a compact phase, such a slow phenomenon simply does not exist: The phase is always instantly delocalized in all wells of the cosine, and moreover, that state is superconducting. The superconducting state observed in these experiments was then the genuine equilibrium state.

\section{APPENDIX B: FABRICATION DETAILS}

The fabrication of the sample starts from a gold $50-\Omega$ coplanar waveguide (CPW) defined by optical lithography and providing the input and output ports for the microwave signals. The central conductor of the transmission line is interrupted on a length of $38 \mu \mathrm{m}$, creating a cavity in which the resistor and junctions are fabricated in two subsequent steps, using $e$-beam lithography and evaporation through suspended masks. The resistor consists of a 8.5-nm-thick, approximately $100-\mathrm{nm}$-wide and $16-\mu \mathrm{m}$-long $\mathrm{Cr}$ wire, periodically overlapped with 45-nm-thick, $1 \times 1(\mu \mathrm{m})^{2} \mathrm{Cr}$ cooling pads. One end of the resistor connects to the output transmission line. The junctions are produced by standard double-angle evaporation of aluminum. The SQUID is connected on one side to the ground plane of the CPW, and on the other side to the other end of the $\mathrm{Cr}$ resistor. Microwave simulations of the circuit are used to check that in this design the actual impedance seen by the junction is close to $R \| C$ up to frequencies well above $(R C)^{-1}$. In order to meet this condition, it is important that the whole SQUID + resistor layout is very compact to avoid stray inductances and capacitances.

\section{Determination of the sample parameters}

Since the values of $E_{J}$ and $R$ cannot be independently measured directly on the sample, the values reported in Table I come from the room-temperature measurements of the resistance of several other junctions and resistors having the same dimensions and fabricated at the same time on the sample. From the scatter of these measurements, the values reported are believed to be accurate within $\pm 15 \%$. The value of $E_{c}$ is estimated from the area of the junction using the commonly used value $100 \mathrm{fF}(\mu \mathrm{m})^{-2}$ for the capacitance per unit area of aluminum-aluminum oxide junctions. The value of the coupling capacitance is obtained from microwave simulations.

\section{APPENDIX C: JOULE HEATING IN THE RESISTOR}

Here we show that for the measurements shown in Fig. 4, the Joule power dissipated in the chromium resistor does not substantially raise the electronic temperature. For this, we rely on the analysis of heating in diffusive wires detailed in Ref. [27], where it is assumed that the electron temperature can be well defined locally, i.e., that the thermalization between electrons occurs faster than their diffusion through the wire and that we can neglect the radiative cooling of the wire. In this reference, the diffusive wire is supposed to be connected to two normal-metal reservoirs at both ends, and these reservoirs are supposed to be large enough so that their electronic temperature is equal to the phonon temperature. In our case, on the junction side the $\mathrm{Cr}$ wire is connected to superconducting $\mathrm{Al}$ which blocks any heat exchange at very low temperatures. We can nevertheless obtain the electronic temperature at this point by considering the results of Ref. [27] in the middle of a wire with twice the length, twice the resistance, and twice the dissipated power. 
We first evaluate the maximum Joule power $P_{R}$ dissipated in the $\mathrm{Cr}$ resistor for the measurements performed at the lowest temperature $(13 \mathrm{mK})$ in Fig. 4. This power is proportional to the power $P_{\text {out }}$ at the output of the sample by

$$
P_{R}=\frac{R}{Z_{0}} P_{\text {out }},
$$

where $Z_{0}=50 \Omega$ is the impedance of the microwave circuitry, and

$$
P_{\mathrm{out}}=P_{\mathrm{VNA}} 10^{\left[\left(S_{21}-G\right) / 10\right]},
$$

where $P_{\mathrm{VNA}}$ is the power at the vector network analyzer (VNA) output, $S_{21}$ is the measured transmission of the setup (in $\mathrm{dB}$ ), and $G=+106 \mathrm{~dB}$ the overall gain (in $\mathrm{dB}$ ) of the microwave chain from the sample output to the VNA input. For sample 2, using the maximum value $\operatorname{Max}\left(\left|S_{21}\right|\right)=$ $-50 \mathrm{~dB}, P_{\mathrm{VNA}}=+3 \mathrm{dBm}$, and $R=8 \mathrm{k} \Omega$, this leads to a maximum $P_{R} \simeq 80 \mathrm{aW}$ [for sample 1: $\operatorname{Max}\left(\left|S_{21}\right|\right)=-50 \mathrm{~dB}$, $P_{\mathrm{VNA}}=-4 \mathrm{dBm}$, and $R=12 \mathrm{k} \Omega$ give a maximum $\left.P_{R} \simeq 25 \mathrm{aW}\right]$.

Looking for an upper bound for the electronic temperature, we consider the simple "interacting hot-electron" limit, where electron-phonon interaction in the wire is neglected, so that cooling occurs only through diffusive electronic exchange with the reservoir (here the gold central conductor of the $\mathrm{CPW}$ ). In this limit, the maximum temperature (reached in the middle of the wire in Ref. [27] and at the Cr-Al interface in our case) is

$$
T_{\max }=\sqrt{T_{\mathrm{ph}}^{2}+\frac{3}{4 \pi^{2}}\left(\frac{e}{k_{B}}\right)^{2} 2 R 2 P_{R}},
$$

where $T_{\mathrm{ph}}$ is the phonon temperature in the reservoir. At the lowest temperature $T_{\mathrm{ph}}=13 \mathrm{mK}$, and for the above values this yields

$$
T_{\max } \simeq T_{\mathrm{ph}}+\left\{\begin{array}{l}
1 \mathrm{mK} \text { for sample } 2 \\
0.5 \mathrm{mK} \text { for sample } 1
\end{array}\right.
$$

which sets an upper bound for the electronic temperature of the electromagnetic environment in our experiments. These considerations show that in the entire experimental range, Joule heating of the resistor is negligible.

In the above analysis, the thick intermediate pads incorporated in the wire design (see Fig. 2) play absolutely no role. They are meant to increase electron-phonon coupling, but they are effective only at higher temperature, as we now discuss. At the maximum power dissipated in the resistor, we can estimate the electronic temperature $T_{\Sigma}=\left(P_{R}^{2} / \Sigma \Omega\right)^{1 / 5}$ [27] that would be reached if only electron-phonon cooling was taking place. Taking the entire volume of the resistive wire and of the intermediate cooling pads $\Omega \simeq 0.20(\mu \mathrm{m})^{3}$ and assuming the standard

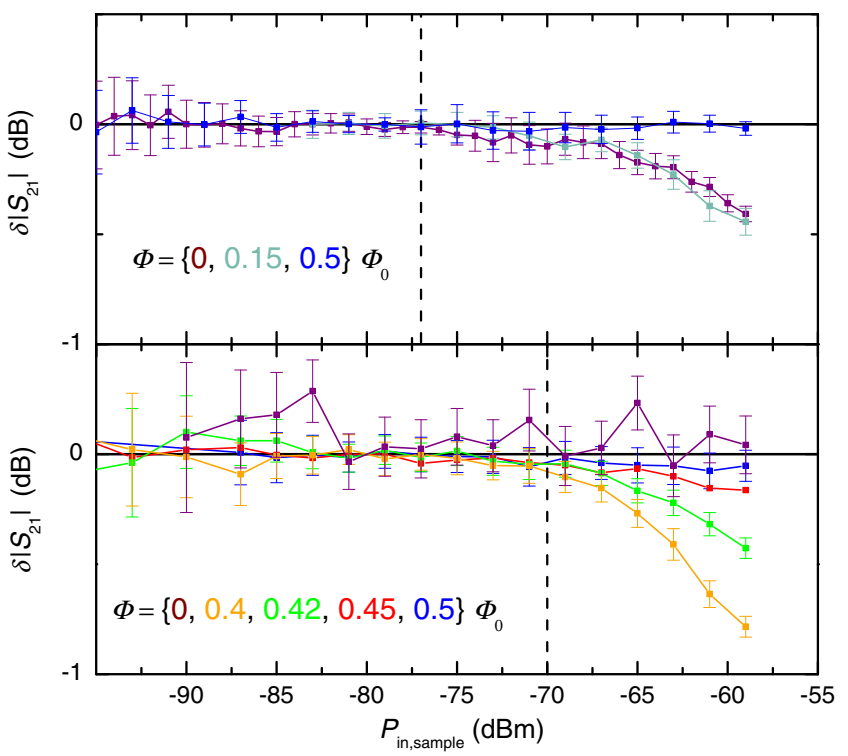

FIG. 5. Variations of the transmission through the samples (top, sample 1; bottom, sample 2), as a function of the power at the sample input, for different values of the flux through the SQUIDs, at the lowest temperature (variations are taken with respect to the value at $P_{\text {in }}=-80 \mathrm{dBm}$ ). The size of the error bars does not vary monotonically because the averaging time was increased when reducing the power. The dashed lines indicate the power levels that were chosen to take the data shown in Fig. 3.

electron-phonon coupling constant $\Sigma \simeq 2 \mathrm{nW}(\mu \mathrm{m})^{-3} \mathrm{~K}^{-5}$ gives $T_{\Sigma} \simeq 36 \mathrm{mK}$ (for sample 2). We could thus increase the measurement power at temperatures above $50 \mathrm{mK}$ in order to speed up the measurements while still not heating the electrons.

\section{APPENDIX D: CHECKING THE LINEARITY OF THE RESPONSE}

In order to ascertain that we measure the linear response of the junction properly, we check that $S_{21}$ no longer depends on applied power at low power. In Fig. 5, we show the variations of $\left|S_{21}\right|$ as a function of the applied measurement power for various fluxes in the two samples at the lowest temperature $(13 \mathrm{mK})$. We indeed observe that in the low-power range, $\left|S_{21}\right|$ no longer changes, confirming that we measure the linear response and that we are not heating the resistor. We used such measurements to choose the operating power for the data presented in Fig. 3, selecting the value at the end of the horizontal plateau (shown as the dashed vertical line in Fig. 5), i.e., $-77 \mathrm{dBm}$ for sample 1 and $-70 \mathrm{dBm}$ for sample 2 .

\section{APPENDIX E: LINK BETWEEN THE MEASURED $S_{21}$ AND THE JOSEPHSON-JUNCTION PARAMETERS}

Assuming a probe signal has a low enough amplitude, the circuit between the input port and the output port of 


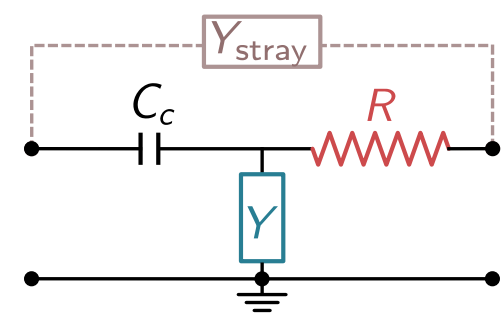

FIG. 6. Quadrupole model of the on-chip components for the calculation of the transmission $S_{21}$. In the ideal case where the external circuit can be fully calibrated by measuring reference samples, $S_{21}$ would depend only on $C_{c}, R$, and $Y$, the admittance of the junction. In our experiment, this full calibration is not performed, and a weak stray admittance $\left|Y_{\text {stray }}\right| \ll\left|i C_{c} \omega\right|$ very likely dominates our measurements at lower values of $S_{21}$, when the junction admittance is large $(|Y| R \gg 1)$.

the sample chip is then a linear quadrupole as depicted in Fig. 6, with the junction described as a linear admittance $Y(\omega)$ corresponding to the parallel combination of the capacitance and the SQUID of Fig. 2. For such a quadrupole, the input and output waves' amplitudes at both ports (assumed to have the standard microwave characteristic impedance $Z_{0}=50 \Omega$ ) are related by an $S$ matrix [49]. Considering that at the frequency of the experiment $\left(i C_{c} \omega\right)^{-1} \gg R \gg Z_{0}$, the $S$ matrix of the sample is approximately

$$
S \simeq I+S_{21} \sigma_{x},
$$

where $I$ is the $2 \times 2$ identity matrix, $\sigma_{x}$ the Pauli matrix, and

$$
S_{21}=\frac{2 i C_{c} \omega Z_{0}}{1+R Y(\omega)}
$$

is the transmission amplitude from the input to the output port.

Thus, in principle, a measurement of the (complexvalued) transmission $S_{21}$ with a vector network analyzer can give access to the complex junction admittance. However, in order to access this ideal on-chip $S$, one must carefully calibrate the whole microwave setup using several reference devices (e.g., thru, reflect, and line) in place of the sample [49] in order to deembed the effect of the rest of the setup. Such a procedure is needed, in particular, to define a reference for $\arg S_{21}$ and to cancel any stray transmission between input and output ( $Y_{\text {stray }}$ in Fig. 6). As our demonstration involves evidencing only a SQUID modulation that saturates at low temperature, it requires only qualitative measurements, and thus, for simplicity, such a calibration is not performed. The measured (uncalibrated) $\left|S_{21}\right|$ variations can nevertheless be qualitatively compared to the ideal prediction

$$
\left|S_{21}\right|^{2}=\frac{\left(2 C_{c} \omega Z_{0}\right)^{2}}{|1+R Y(\omega)|^{2}} .
$$

Given our choice of parameters $R C \omega \ll 1$, the capacitive contribution in $Y$ can be neglected for the evaluation of $S_{21}$, and we can consider only the contribution of the Josephson element $Y(\omega) \simeq 1 / i L_{\text {eff }} \omega$ (assuming a superconducting character). Under this form, it is clear that larger values of $\left|S_{21}\right|$ correspond to small junction admittance (large effective inductance, small supercurrent) and vice versa.

For sample 1, in the low-temperature limit, the modulation of $\left|S_{21}\right|$ with flux is small, showing that $|Y| R \ll 1$. Assuming the junction behaves as a usual symmetric SQUID, its inductance depends on the flux $\Phi$ as $L_{\text {eff }}(\Phi)^{-1}=2 e I_{0}^{\text {eff }}\left|\cos \left(\pi \Phi / \Phi_{0}\right)\right| / \hbar$, with $I_{0}^{\text {eff }}$ the effective critical current. By adjusting the amplitude of the $S_{21}$ modulation for sample 1 in the low-temperature limit, this gives $I_{0}^{\text {eff }} \sim 70 \mathrm{pA}$, much smaller than the AmbegaokarBaratoff $I_{0}=E_{J} 2 e / \hbar=5.0 \mathrm{nA}$ value obtained from the junction's tunnel resistance. This decrease is qualitatively expected, because zero-point phase fluctuations [23,50,51] are known to reduce the effective critical current, or, equivalently, to "renormalize" the apparent Josephson coupling. One can also check that the change of $\left|S_{21}\right|$ of approximately $-1.4 \mathrm{~dB}$ between the maximum frustration of the SQUID at low temperature (where $Y \simeq 0$ ) and the critical temperature $T_{c} \sim 1.2 \mathrm{~K}$ of $\mathrm{Al}$ (where all lines merge at $Y \simeq 1 / R_{T}$ ) is consistent with $R_{T} \simeq 62 \mathrm{k} \Omega$, the junction normal-state tunnel resistance. This line of reasoning is also true for sample 2: The change of $\left|S_{21}\right|$ of approximately $-3.6 \mathrm{~dB}$ is consistent with $R_{T} \simeq 19 \mathrm{k} \Omega$.

For sample 2, however, it is not possible to correctly reproduce the shape of the variations of $S_{21}$ in the bottom left panel of Fig. 3 by assuming the SQUID behaves as a standard one with $L_{\text {eff }}(\Phi)^{-1}=2 e I_{0}^{\text {eff }}\left|\cos \left(\pi \Phi / \Phi_{0}\right)\right| / \hbar$ and adjusting the effective $I_{0}^{\text {eff }}$ as done for sample 1 . Given the shape of the modulation, it seems very likely that a weak stray transmission in our setup (as $Y_{\text {stray }}$ in Fig. 6) causes $\left|S_{21}\right|$ to saturate at a minimum value of approximately $-65.7 \mathrm{~dB}$. Note that even if this were not the case, we expect the modulation curve could still not be accurately fitted using $L_{\mathrm{eff}}(\Phi)^{-1}=2 e I_{0}^{\mathrm{eff}}\left|\cos \left(\pi \Phi / \Phi_{0}\right)\right| / \hbar$, because in this sample, the $\left|S_{21}\right|$ measurements show that the junction admittance is modulated from $|Y| \lesssim$ or $\ll 1 / R$ at the maximum frustration to $|Y| \gg 1 / R$ at minimum frustration, such that the total effective impedance at the junction and the corresponding zero-point phase fluctuations (which determine $I_{0}^{\text {eff }}$ ) vary much with $\Phi$. This variation of admittance should lead to a strongly flux-dependent $I_{0}^{\text {eff }}(\Phi)$, and hence, an overall non-abs(cos) modulation of the inverse inductance [23].

Finally, the striking nonmonotonic dependence of the transmission on the temperature is explained qualitatively in the main text before the Conclusion.

Our experiment demonstrates that when quantitative predictions become available for the junction inductance in high-impedance Ohmic environments, calibrated $S_{21}$ 
measurement in such a setup should allow a quantitative comparison.

\section{APPENDIX F: LINEAR RESPONSE AND MOBILITY IN THE CALDEIRA-LEGGETT MODEL}

Considering the Hamiltonian $H^{\prime}$ of the main text

$$
H^{\prime}=E_{C}\left(N-N_{R}\right)^{2}-E_{J} \cos \varphi+\sum_{n} 4 e^{2} \frac{N_{n}^{2}}{2 C_{n}}+\frac{\hbar^{2}}{4 e^{2}} \frac{\varphi_{n}^{2}}{2 L_{n}},
$$

the operator for the current flowing through the junction is

$$
I=\frac{2 e}{\hbar} \frac{\partial H^{\prime}}{\partial \varphi}=\frac{2 e}{\hbar} E_{J} \sin \varphi=I_{0} \sin \varphi .
$$

Now we consider a thought experiment where the junction phase $\varphi$ is given a time dependence $\varphi \rightarrow \varphi+\delta \varphi(t)$, so that the Hamiltonian acquires a time dependence too, $H^{\prime} \rightarrow H^{\prime}(t)$. We can obtain the corresponding change in the current by using the general response formula of Ref. [52] [Eq. (1) with $X\left(t^{\prime}\right) \equiv \varphi\left(t^{\prime}\right)$ and $\hat{O}(t) \equiv I(t)$ ],

$$
\begin{aligned}
\frac{\delta I(t)}{\delta \varphi\left(t^{\prime}\right)}= & \frac{-i}{\hbar} \theta\left(t-t^{\prime}\right)\left\langle\left[I(t), \frac{\hbar}{2 e} I\left(t^{\prime}\right)\right]\right\rangle \\
& +\delta\left(t-t^{\prime}\right) \frac{2 e}{\hbar} E_{J}\langle\cos \varphi\rangle(t),
\end{aligned}
$$

where $\langle\ldots\rangle=\operatorname{tr}[\rho(t) \ldots]$ with the time-dependent density matrix $\rho(t)$. This result expresses the exact Hamiltonian evolution, making essentially no assumption on the system or on the drive $\delta \varphi(t)$. However, it involves the timedependent density matrix $\rho(t)$. The linear response of the system is obtained from this general formula by considering vanishingly small $\delta \varphi(t)$, in which case, we can use the equilibrium density matrix in the above expression (with the last term becoming time independent and the first one depending only on $t-t^{\prime}$ ).

Using the fact that the voltage fluctuations across the junction are $\delta V=(\hbar / 2 e)(d / d t)[\delta \varphi(t)]$, and going to the frequency domain, the above result yields the junction's linear admittance

$$
\begin{aligned}
Y(\omega)= & \frac{\delta I(\omega)}{\delta V(\omega)}=\frac{1}{\hbar} \int_{0}^{+\infty} 2\langle[I(t), I(0)]\rangle \frac{e^{i \omega t}-1}{\omega} \frac{d t}{2 \pi} \\
& +\left(\frac{2 e}{\hbar}\right)^{2} \frac{E_{J}\langle\cos \varphi\rangle}{i \omega} .
\end{aligned}
$$

In the first term, one recognizes the standard linear susceptibility of the usual Kubo formula. The second term is due to the (change in the) current carried by the ground state, yielding a purely inductive response of the junction. Even if we do not know what the equilibrium density matrix is in our system (because the junction is entangled with the bath), this term is nonzero as long as $E_{J}\langle\cos \varphi\rangle \neq 0$.

Using the fact that $2 e \dot{N}=I$, the first term in the above expression can also be formulated in terms of the junction's charge correlator

$$
\begin{aligned}
Y(\omega)= & \frac{4 e^{2}}{\hbar} \int_{0}^{+\infty} 2\langle[N(t), N(0)]\rangle \omega e^{i \omega t} \frac{d t}{2 \pi} \\
& +\left(\frac{2 e}{\hbar}\right)^{2} \frac{E_{J}\langle\cos \varphi\rangle}{i \omega} .
\end{aligned}
$$

The impedance defined by this thought experiment is an equilibrium property of the junction coupled to its environment. In practice, when one wants to measure this linear response, indirect driving of the junction phase can be realized in several ways, say, by threading an ac magnetic field in the circuit loop or by using a capacitive bias as in our experimental setup. As long as the probing circuitry does not alter the impedance seen by the junction, the measured linear response is (and it must be) independent of the biasing scheme chosen. We further stress that the linear response theory naturally embraces finite frequencies so that $Y(\omega)$ is a genuine equilibrium property of the system, even at finite frequency. In this regard, our probing of the system at approximately $1 \mathrm{GHz}$ poses no problem of principle.

\section{1. dc mobility vs full linear response}

In the entire literature on the Schmid-Bulgadaev transition, the transport quantity that was focused on is the so-called dc charge mobility,

$$
\mu=\frac{\delta I_{\mathrm{dc}}}{\delta V_{\mathrm{dc}}}=\operatorname{Re} Y(\omega=0),
$$

which entirely comes from the first term of the admittance (F1) and that is obtained considering only the equilibrium charge (or current) correlator. Note that, by definition, $\mu$ describes dissipative transport.

However, if the inductive term in $Y(\omega)$ is nonzero (i.e., if the system can sustain a supercurrent), the zerofrequency limit of Eq. (F1) considered in $\mu$ is disregarding a diverging term, and, given that there are never strictly zero-frequency measurements, one may wonder about the relevance of this quantity for describing transport. Indeed, we now show that no experimental measurement protocol gives access to $\mu$ in a superconducting system. Let us, for instance, consider the initial unbiased equilibrium state with $\langle\varphi\rangle=0$ in which there is no current flowing $[I(t \leq 0)=0]$ and assume that at $t=0$, a voltage step $\delta V(t)$ is applied, ending on a plateau $\delta V \neq 0$ after a time $\tau$. If mobility were appropriately describing the linear dc response, one would expect that after a transient, $\delta I(t \gg \tau) \rightarrow \mu \delta V$. However, this is clearly not the case for a linear superconducting inductor because the inductive response to the voltage pulse is a linearly 
increasing current, not a transient. It is also incorrect for a Josephson junction and its nonlinear inductance because the interplay of the Josephson nonlinearity and the non-Markovianity due to the retarded response of the $R C$ circuit results in a complex dynamics of the system involving potentially many harmonics of the Josephson frequency $\omega_{J}=2 e \delta V / \hbar$. There is presently no general theory that is able to predict the resulting dc current for all parameters and, in particular, when phase fluctuations are large. Here we assume a voltage bias scheme, but one can similarly show that the inductive response cannot be ignored in other biasing schemes and that this cannot be fixed by changing the frequency, the amplitude, or the temperature at which the measurement would be performed. In a nutshell, the linear mobility simply does not properly describe transport in a system that can sustain a supercurrent (i.e., where $E_{J}\langle\cos \varphi\rangle \neq 0$ in the case we consider) because it ignores the dominant effect of the supercurrent.

Consequently, finding a vanishing dc mobility (as in Refs. $[1-6,9,11,12])$ is not by itself a correct way of proving the system is insulating. For being an acceptable proof, it requires, in addition, that the coherences are suppressed in the ground state. Note that interestingly, Schmid [1] also considered the renormalization of the coherence factor $E_{J}\langle\cos \varphi\rangle$ (see the following section), but he regarded this as an independent proof of his mobility result, and not a condition for it.

\section{Insulating state in the Caldeira-Leggett model?}

From the above material, it emerges that for an insulating state to exist in this Caldeira-Leggett model, it is necessary (and sufficient) that the environment fully suppresses the coherences between charge states in the ground state.

As explained in the main text, RG flow analysis on the Josephson coupling initially indicated that coherences also vanished in the insulating phase, seemingly validating the mobility calculation (on the insulating side). However, results on the spin-boson problem, as well as perturbation theory in $E_{J}$ in the CL model contradict the RG analysis and indicate that finite coherences always survive in the ground state of the CL model (as long as $E_{J} / E_{C}>0$ ). Hence, from the theory point of view, it is clear by now that within the CL model, a dissipative environment can reduce the coherence only to a certain point. So, a remaining finite supercurrent is to be expected, and that is indeed what we and Grimm et al. [29] observe. Beyond our experiment, this behavior is expected for the entire parameter space: The junction is superconducting everywhere. This resolves the conflicts evoked in the Introduction.

Note that our conclusion that mobility calculations do not correctly describe transport in the CL model (and therefore cannot be used to predict a superconductinginsulating transition) is independent of whether one considers a compact or extended phase description; it applies also to old works which explicitly considered an extended phase for evaluating the mobility. The compact phase symmetry put forward by our analysis is still very important because it enables us to reach a simple consistent picture in all known limits, and, through the self-duality of the model, it clarifies how decompactification occurs.

\section{APPENDIX G: THE PREDICTED PHASE TRANSITION IN THE $P(E)$ THEORY}

In Ref. [4], Aslangul et al. use a tight-binding model to describe junctions coupled to a linear environment in the so-called scaling limit, and they confirm Schmid's prediction of a phase transition. Here we go over their derivation using the notations more commonly used at present for Josephson circuits.

First we express the Hamiltonian $\tilde{H}$ considered in Ref. [4] as

$$
\tilde{H}=\frac{E_{J}}{2}\left(\sum_{N \in \mathbb{Z}}|N\rangle\langle N+1| e^{i \tilde{\varphi}+2 i e V t / \hbar}+\text { H.c. }\right)+H_{\text {bath }},
$$

where $N$ is the number of charges passed through the junction, and $\tilde{\varphi}$ is the fluctuating phase across the (disconnected) environment (in the notations of Ref. [4], $\left.B_{+}=e^{i \tilde{\varphi}}, \hbar \Delta=E_{J}\right)$. For more generality, we consider the case where a voltage source is present (the results of Ref. [4] are recovered taking $V=0$ ). This Hamiltonian is also considered in Ref. [53]. Note that when using this tight-binding description of discrete charge states, it implies the junction phase is considered compact.

The current operator through the junction is

$$
\hat{I}=i \frac{2 e}{\hbar} \frac{E_{J}}{2}\left(\sum_{N \in \mathbb{Z}}|N\rangle\langle N+1| e^{i \tilde{\varphi}+2 i e V t / \hbar}-\text { H.c. }\right) .
$$

We now evaluate the current correlator

$$
S_{I I}(t)=\langle\hat{I}(t) \hat{I}(0)\rangle,
$$

assuming that the backaction of the junction on the environment is weak enough to not modify the equilibrium properties of the bath. At first view, this assumption can be justified if the junction impedance at its plasma oscillation is much larger than the environment resistance (i.e., $\sqrt{E_{C} / E_{J}} \gg R / R_{Q}$ ), in which case the environment imposes its phase fluctuations onto the junction. This condition is indeed fulfilled in the scaling limit considered in Ref. [4]. Within these hypotheses, the correlator evaluates to 


$$
\begin{aligned}
S_{I I}(t)= & \left(\frac{2 e}{\hbar} \frac{E_{J}}{2}\right)^{2}\left[\left(\sum_{N \in \mathbb{Z}}\langle N|\rho| N\rangle\right)\left(\left\langle e^{i \tilde{\varphi}(t)} e^{-i \tilde{\varphi}(0)}\right\rangle e^{2 i e V t / \hbar}+\left\langle e^{-i \tilde{\varphi}(t)} e^{i \tilde{\varphi}(0)}\right\rangle e^{-2 i e V t / \hbar}\right)\right. \\
& \left.-\left(\sum_{N \in \mathbb{Z}}\langle N|\rho| N+2\rangle\left\langle e^{i \tilde{\varphi}(t)} e^{i \tilde{\varphi}(0)}\right\rangle e^{2 i e V t / \hbar}+\langle N|\rho| N-2\rangle\left\langle e^{-i \tilde{\varphi}(t)} e^{-i \tilde{\varphi}(0)}\right\rangle e^{-2 i e V t / \hbar}\right)\right],
\end{aligned}
$$

where $\rho$ is the reduced density matrix of the junction. Considering that the linear environment remains in equilibrium, its fluctuations are Gaussian, and one has

$$
\left\langle e^{ \pm i \tilde{\varphi}(t)} e^{\mp i \tilde{\varphi}(0)}\right\rangle=e^{J(t)}
$$

and

$$
\left\langle e^{ \pm i \tilde{\varphi}(t)} e^{ \pm i \tilde{\varphi}(0)}\right\rangle=e^{-J(t)+2 J(\infty)}
$$

with

$$
\begin{aligned}
J(t) & =\langle[\tilde{\varphi}(t)-\tilde{\varphi}(0)] \tilde{\varphi}(0)\rangle \\
& =\int_{-\infty}^{+\infty} \frac{d \omega}{\omega} \frac{\operatorname{Re} Z(\omega)}{2 R_{Q}} \frac{e^{-i \omega t}-1}{1-e^{-\beta \hbar \omega}},
\end{aligned}
$$

with $Z$ being the total environment admittance as seen from the Josephson element, including the junction capacitance [i.e., $Z(\omega)=\left(R^{-1}+i C \omega\right)^{-1}$ ]. For an Ohmic environment $\operatorname{Re} J(\infty)=-\infty$, so that the terms in the second line of Eq. (G1) vanish, and using $\operatorname{tr} \rho=1$, the correlator finally reduces to

$$
S_{I I}(t)=\left(\frac{2 e}{\hbar} \frac{E_{J}}{2}\right)^{2} e^{J(t)} \cos \frac{2 e V t}{\hbar},
$$

or, in the frequency domain,

$$
S_{I I}(\omega)=\left(\frac{2 e}{\hbar} \frac{E_{J}}{2}\right)^{2}[P(\hbar \omega+2 e V)+P(\hbar \omega-2 e V)],
$$

where

$$
P(E)=\frac{1}{2 \pi \hbar} \int_{-\infty}^{\infty} \exp [J(t)+i E t / \hbar] d t
$$

is the usual $P(E)$ function considered in dynamical Coulomb blockade. For the $R C$ environment considered here, at zero temperature one has [53]

$$
P(E) \propto E^{2 R / R_{Q}-1} .
$$

\section{Charge transport}

The standard Green-Kubo relations link the admittance $Y_{G K}$ to $S_{I I}$,

$$
\operatorname{Re} Y_{G K}(\omega, V)=\frac{1}{2 \omega}\left[S_{I I}(\omega)-S_{I I}(-\omega)\right] .
$$

Note that, even after applying Kramers-Kronig relations to get the imaginary part, this admittance $Y_{G K}$ corresponds only to the first term in Eq. (F1) and therefore lacks the inductive response of the junction, which we know is important (see Appendix F). If we nevertheless proceed, from Eq. (G3) one predicts a differential conductance

$$
\begin{aligned}
\frac{d I}{d V}(V) & =\operatorname{Re} Y_{G K}(\omega \rightarrow 0, V) \\
& =\frac{2 e^{2}}{\hbar} E_{J}^{2}\left[P^{\prime}(2 e V)+P^{\prime}(-2 e V)\right],
\end{aligned}
$$

and the $I-V$ characteristics are obtained by straightforward integration

$$
I(V)=\frac{e}{\hbar} E_{J}^{2}[P(2 e V)+P(-2 e V)] .
$$

The above results are already found in Ref. [53]. They describe inelastic tunneling processes of Cooper pairs with real transitions in the environment modes. The $I-V$ characteristic (G4) is known to quantitatively describe experiments $[50,54]$ at finite voltages when the Josephson coupling is small enough that the environment modes remain in equilibrium.

From the above results, one predicts the junction zerobias conductance

$$
\begin{aligned}
G & =Y_{\mathrm{GK}}(\omega \rightarrow 0, V=0) \\
& =\frac{2 e}{\hbar} E_{J}^{2} P^{\prime}(0),
\end{aligned}
$$

which corresponds to the dc charge mobility calculated by Aslangul et al. [4]. Thus, for the $R C$ environment considered here, at $T=0$, using Eqs. (G2) and (G5), one recovers the "superconducting-to-insulating" phase transition at $R=R_{Q}$, as found by Aslangul et al. [4] (Schmid [1] and Bulgadaev [2] obtained the same results for the mobility by mapping the problem onto a log-gas). However, as noted above, the charge-transfer processes described here are inelastic, and it is therefore not correct to describe this type of process as superconducting transport for $R<R_{Q}$.

\section{Conclusions on the phase transition}

(i) As shown in Appendix F, $Y_{\mathrm{GK}}$ is not the full linear admittance; it entirely misses the inductive response 
of the junction and cannot properly describe charge transport (and notably the supercurrent branch in the $I-V$ characteristics) because of that. This perturbative tight-binding approach does predict a transition in the charge correlator (a partial charge localization), but it is incorrect to infer from this result that a superconducting-insulating transition exists.

(ii) The predicted transition in the junction charge correlator arises entirely from the $P(E)$ function, i.e., from the equilibrium fluctuations across the $R C$ environmental impedance not connected to anything. It has nothing to do with the junction. This is already noted in Ref. [3].

(iii) As we mention in the main text, for $R<R_{Q}$, the charge is predicted to be fully delocalized, and correspondingly, the junction phase is fluctuationless (and its dynamics is that of a classical quantity).

The last two points seem odd and most likely too sketchy. Just as it is now understood that the coherence factors do not actually vanish in this system, it is quite clear that taking into account the backaction of the junction on the environment (causing their entanglement) would suppress the above sharp transition in the charge correlator and turn it into a smooth crossover with finite but small phase (resp. charge) fluctuations in the (resp. dual of the) delocalized charge state. Actually, we know this is the expected behavior when $E_{J} \gg E_{C}$ and $R \gg R_{Q}$ [upper left corner of the diagram in Fig. 4(b)]: At low temperatures, such a junction behaves essentially as a linear inductor, and it is well known that parallel $R L C$ circuits have finite charge and phase fluctuations for all parameters. Then, using the duality argument, the presence of finite charge and phase fluctuations should also be true for $E_{J} \ll E_{C}$ and $R<R_{Q}$. Finally, by continuity, this should be also true in the entire diagram.

The continuous crossover that emerges from our analysis contrasts with the results of the Monte Carlo simulations performed assuming an extended phase in Ref. [11], where an abrupt transition in the phase correlator at $T=0$ and $R=R_{Q}$ is found. This discrepancy illustrates that considering an extended phase can lead to results inconsistent with our analysis (see Appendix H).

In conclusion, we expect no DQPT transition in the CL model: neither a superconducting-insulating transition nor a transition in the charge or phase correlator.

\section{APPENDIX H: COMPACT VS EXTENDED JUNCTION PHASE}

The analysis of the CL model conducted in the main text is based on symmetry considerations and leads to a "phase diagram" [Fig. 4(b)], which is theoretically consistent (including at its boundaries) and consistent with experiments. In this phase diagram, the junction phase is compact below the antidiagonal and it progressively decompactifies above the antidiagonal in a smooth crossover.
We stress that when this decompactification does not occur, compact phase solutions are dictated by the symmetry of (the effective Josephson Hamiltonian in) the CL model; this symmetry is not for the physicist to choose. As a corrollary, choosing to use an extended phase in many earlier works on the CL model cannot be rigorously justified theoretically because there are no known mechanisms within that model that would break the system's fundamental symmetry in this way. The only established symmetry breaking for the phase is the partial decompactification we describe in the main text (but it cannot be found starting from an extended phase).

Since it cannot be justified within the model, making use of an extended phase implicitly and forcefully adds poorly controlled hypotheses or ingredients to the model, with essentially unknown consequences. (In practice, it adds an additional variable indexing the wells of the cosine and enabling us to distinguish all of them in all circumstances, which is not possible in the original model.) For sure, this can be done theoretically-it works. But does such a treatment still yield fully relevant predictions for the realworld system that the model was originally meant to describe? Clearly not. It is certain that predictions will differ in circumstances where interferences between the wells matter, and this difference is unavoidable in a system with superconducting coherences such as the one we consider. In other words, in the parameter space where we now know the phase discrete translational symmetry is not spontaneously broken, there exists mathematically correct extended phase solutions for $H$ that cannot be unitarily transformed to a suitable (i.e., compact) solution for $H^{\prime}$. Such solutions do not respect the intrinsic system symmetry, but it is nearly impossible to figure this inconsistency by considering only the extended phase hypothesis.

This subtle point on the junction phase symmetry and its spontaneous breakage has never been properly understood so far. We think that bringing this point up and clarifying it is a significant achievement of the present work.

\section{Retrospective on the compact vs extended phase debate}

Prior to this work, it was intuited that phase decompactification must take place somehow (at least for some parameters), but it was not understood how it was occurring, and this resulted in a lot of ambiguities and confusion. Here, we try to put into perspective why the situation was so confusing.

An extended phase description contains the compact phase solutions as solutions of higher symmetry (periodic solutions in phase representation), so that, in principle, it should be the only description ever needed. Indeed, when starting from a Hamiltonian such as $H$ in the main text, for which an extended phase is the "natural" point of view, one can obtain the compact phase solutions by considering highly nontrivial initial and boundary conditions [33,34]. 
However, in the existing literature based on using $H$, this was not done, and, as a consequence, compact phase solutions (which are of utmost importance as our work shows) were not found or not recognized as such.

Until now, this seemed not too problematic, and it was even rationalized that compact phase states were irrelevant in systems that are most conveniently described using $H$ (essentially, systems where a dc current can flow). The rationale was that in these systems, a "full decompactification" process (i.e., yielding only nonperiodic extended phase states solutions of $H$ ) would always occur for all parameters and all temperatures. At first, this was just argued for qualitatively [55]. Soon after, Zwerger et al. [56] showed that such a full decompactification process should indeed always occur for Ohmic environments, but their derivation can no longer be considered conclusive as it did not take into account the entanglement of the junction with the environment, which we now know is key. Later, Apenko proposed another justification [35], but in his derivation, the identification of different phases in the circuit was not rigorous (similar to what we discuss about the Hamiltonian of the fluxonium circuit in Appendix H 4).

Hence, schematically, for a very long time, it was broadly considered that the symmetry of the phase and the Hamiltonian used were somehow tied: $(H \Leftrightarrow$ extended phase assumed to be a decompactified phase) XOR ( $H^{\prime} \Leftrightarrow$ compact phase).

To support this dichotomic view, several arguments or criteria were used to favor using a compact or an extended phase description, depending on the problem considered. For instance, it was frequently argued that a compact junction phase is suitable only in circuits having an "island" connected to the junction, as it would be a manifestation of the charge quantization in the island or of the tunneling of individual Cooper pairs through the junction. In other words, a compact phase should not be appropriate in a circuit where the charge can flow continuously (and thus, considering $H^{\prime}$ to describe the Ohmic shunted junction was not considered appropriate). Although the general discussion of the main text already shows such arguments are not relevant, in the following subsections of this Appendix we nevertheless specifically discuss why these arguments do not hold.

\section{Phase compactness is not due to the tunneling of individual Cooper pairs through the junction}

If instead of a Josephson junction one considers a superconducting ballistic (or nearly ballistic) weak link, then the current-phase relation is still periodic with the phase, so that one can again use a discrete charge basis to describe the state of the weak link. In that case, this apparent "charge discretization" obviously cannot be directly linked to an underlying charge quantization due to the tunneling of charge carriers.

\section{Is charge quantization due to the presence of islands?}

As we discuss in the main text, using the discrete charge basis of the CPB (equivalent to considering a compact phase) arises from the symmetries of the system. It does not require the presence of "an island" in which the charge is "naturally quantized." The simplest argument against this is that in a CPB the mere presence of the Josephson junction destroys this charge quantization (the ground state of the CPB consists of a coherent superposition of charge states, with finite zero-point fluctuations). This "charge quantization" is not observable, it is only a mathematical illusion, actually.

Our statement is further supported by the fact that the form of the Caldeira-Leggett Hamiltonian is independent of whether the circuit has an island or not. This can be shown using the explicit decomposition of the total circuit impedance into oscillators according to the rules in Ref. [22].

Finally, one can show that the Hamiltonian of a circuit with an island has a smooth limit to the islandless case by taking the limit where the capacitance defining the island becomes infinite. Correspondingly, all the finite-frequency linear response functions of the system have smooth limits too. However, as the system is nonlinear, the linearity range may vanish at low frequency (see, e.g., Appendix A), depending on the type of response probed. This agrees with the obvious expectation that at strictly zero frequency no dc current can flow when there is an island, while it can if there is no island. As we explain in the main text, the absence of dc current in a circuit with an island results from having a single ground state, while there is a continuum of them in the islandless case permitting a dc current flow.

As a conclusion, whether one considers a CPB with an island or a galvanically shunted junction does not radically change the way the system is modeled.

\section{The junction's phase in the fluxonium}

It is frequently argued that one must use an extended phase description for describing the fluxonium circuit [57] where a Josephson junction is connected in parallel with a inductor (instead of a resistor in this paper).

Indeed, for the fluxonium, the Hamiltonian proposed in Refs. [25,57] is

$$
H_{f 1}=\frac{q^{2}}{2 C}-E_{J} \cos \varphi+\frac{\left(\Phi_{\mathrm{ext}}-\frac{\hbar}{2 e} \varphi\right)^{2}}{2 L},
$$

where $(\hbar / 2 e) \varphi$ and $q$ denote the branch flux and charge of the junction, and $\Phi_{\text {ext }}$ is the magnetic flux enclosed by the loop formed between the junction and the inductor considered as an external control parameter, i.e., a fixed real number. In this model, obviously not invariant upon $\varphi \rightarrow \varphi+2 \pi$, the junction's phase clearly appears as extended. However, the eigenstates of the system have current fluctuations that, in addition to vacuum flux 
fluctuations, cause fluctuations of the flux in the loop, which contradicts the assumption that $\Phi_{\text {ext }}$ is a fixed parameter. Thus, the model is not fully consistent.

Another fluxonium Hamiltonian is derived in Ref. [45]. It reads

$$
H_{f 2}=\frac{(Q+q)^{2}}{2 C}-E_{J} \cos \varphi+\frac{\Phi^{2}}{2 L} .
$$

In this writing, $\Phi$ and $Q$ denote the branch flux and charge of the inductor, while $(\hbar / 2 e) \varphi$ and $q$ still denote the branch flux and charge of the junction. This Hamiltonian thus has 2 quantum degrees of freedom (each with fluctuations), and the flux in the loop is given by Kirchhoff's law

$$
\frac{\hbar}{2 e} \varphi-\Phi=\Phi_{\text {loop }}
$$

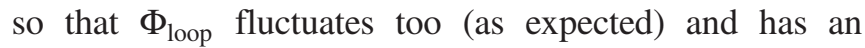
expectation value related to the externally applied flux $\Phi_{\text {ext }}$. It is only by suppressing one of the quantum degrees of freedom, turning it into a classical one, that Eq. $(\mathrm{H} 2)$ becomes Eq. (H1) (and, strictly, $\varphi$ can no longer be considered as a degree of freedom describing the sole junction). The junction's phase appearing as extended in Eq. (H1) thus results from an approximation (perhaps a very good one); it is not an obligation.

The inconsistency pointed out above is a general problem of the circuit quantization scheme proposed in Ref. [22], where loop fluxes are always assumed constant. It can be easily fixed though. Other quantization schemes have also been proposed $[45,58,59]$ which do not necessarily force this approximation.

\section{a. The fluxonium is not in the phase diagram}

In the fluxonium circuit, the impedance seen by the junction has $\operatorname{Re} Z(\omega=0)=0$, which would naively locate it on the right axis of the SB phase diagram. However, in that limit, the system considered in the main text is illdefined as neither the loop inductance $L$ (which defines a new energy scale $E_{L}=\hbar^{2} / 8 e^{2} L$ in the problem) nor the external flux $\Phi_{\text {ext }}$ threading the loop are specified. Thus, the phase diagram would need to be refined with extra parameters close to the right axis.

Nevertheless, depending on its parameters, we expect the fluxonium's junction phase will evolve between fully decompactified (in a single well of the cosine) when $E_{L} \gg E_{J}$ and $\Phi_{\text {ext }} \bmod \Phi_{0} \neq \frac{1}{2}$, partially decompactified (in several wells) when $E_{L} \sim E_{J}$, and essentially compact (populating many wells nearly equally) [25] when $E_{L} \rightarrow 0$.

\section{Phase in current-biased junctions}

When considering the case of a current-biased junction, where the current source "tilts the washboard potential," the different wells of the cosine appear as nonequivalent.
Here again, the obligation to use an extended phase is only apparent.

First, the current source can be modeled by considering a very large inductor loaded with an initial flux. So we are back to considering the fluxonium case for which we argue above that there is no obligation to use an extended phase.

One can arrive at a similar conclusion by performing a time-dependent unitary transformation [34] that removes the tilt of the washboard, restoring the periodicity of the cosine potential. In this case, however, the states of the system will be time dependent.

In such a current-biased junction, the final degree of phase decompactification will depend on the dissipation in the system and on the ratio $E_{J} / E_{C}$ (as in the unbiased case) but certainly also on the current bias $I_{b}$ which sets an extra energy scale $I_{b} \Phi_{0}$ in the system, with an associated dynamics.

\section{APPENDIX I: VALIDITY OF THE EFFECTIVE JOSEPHSON HAMILTONIAN AND CONSISTENCY OF THE CALDEIRA-LEGGETT MODEL}

In the CL model, the junction is modeled using the effective Josephson Hamiltonian (i.e., the celebrated washboard potential for the junction phase) which describes only Cooper pair tunneling, and one couples this effective Hamiltonian to the linear environment.

This effective Josephson Hamiltonian emerges from the tunneling of quasiparticles at second order in perturbation theory in the absence of an environment [60,61], and it is commonly admitted it describes well a junction at energies much lower than the superconducting gap $\Delta$ and in the absence of quasiparticles (which is expected at $k_{B} T \ll 2 \Delta$ ). Even when these conditions are fulfilled, one may wonder whether considering the effect of the environment on this effective Hamiltonian - as done in the CL model - is fully consistent.

A more rigorous and consistent way of considering the effect of the environment on the junction consists of going back to the tunneling of quasiparticles $[23,62,63]$. Doing so, one however finds that at second order in tunneling (corresponding to the effective Josephson Hamiltonian used in $H$ or $H^{\prime}$ ), the junction sees the bare zero-point fluctuations of the $R C$ circuit. However, at that lowest order in perturbation, phase fluctuations are divergent for any Ohmic environment, and this divergence predicts a complete suppression of the supercurrent at all temperatures [23], even for $R<R_{Q}$, in the phase where a classical compact phase is predicted. This shows that the CL description of the system (using $H$ or $H^{\prime}$ ) is inconsistent when considering an Ohmic environment. These inconsistencies resolve at higher orders in the tunneling Hamiltonian (or using a self-consistent approximation [23]), when the inductive backaction of the junction on the environment is taken into account: The junction and 
environment become entangled, voltage and phase fluctuations are reduced, and they acquire an effective superOhmic spectral density for which no DQPT is expected, the junction preserving a finite supercurrent at $T=0$ for all environmental impedances.

Our present work shows that even within the CL model (although it is not fully consistent), the predicted phase transition similarly disappears when considering the backaction of the junction on the environment.

\section{APPENDIX J: RELATIONSHIP WITH PHASE TRANSITIONS IN OTHER SYSTEMS}

The phase transition predicted by SB is closely related to a number of other phase transitions predicted in different systems (see Ref. [64]).

In particular, it is related to the impurity-induced transition in a $1 \mathrm{D}$ conducting channel of interacting spinless fermions (i.e., a Tomonaga-Luttinger liquid, TLL) predicted by Kane and Fisher [65] (KF), according to which, at $T=0$, for any nonzero strength of the impurity potential, the channel conductance should vanish for repulsive interactions $g<1$, while it should reach the perfect TLL conductance $g e^{2} / h$ for attractive interaction $g>1$. This behavior is akin to the SB prediction of a superconducting-to-insulating transition. Kane and Fisher showed that these systems are indeed described by the same effective action, and, according to the principle "the same equations have the same solutions" made famous by Feynman, no one questioned they would have the same phase transition physics until now, even when it became evident that the SB prediction conflicted with known results on Josephson junctions.

\section{Confirmations of the KF phase transition}

Repulsive Luttinger liquids with rational values of $g<1$ have been extensively studied theoretically since they notably describe the low-energy physics of fractional quantum Hall edge states [66]. Thanks to the methods of integrable systems, exact results have been obtained for the specific values of $g=1 / 2$ [67], $g=1 / 3$ [68], and $g=2 / 3$ [69]. All these results corroborate the perturbative RG analysis [65] predicting universal scaling laws for the dc conductance which drive the system to an insulating state as the temperature is lowered for all impurity backscattering strength.

The KF phase transition physics was confirmed experimentally by taking advantage of a second mapping put forward by Safi and Saleur [70], who noticed the action of an impurity in a TLL is also equivalent to that of a singlechannel quantum point contact in series with a resistor $(\mathrm{QPC}+R)$. In this mapping, the TLL interaction parameter $g$ is controlled by the resistance $g=1 /\left(1+R / 4 R_{Q}\right)$, and thus covers only the dynamics of repulsive TLLs. Since the physical implementation of QPC $+R$ is much better controlled than that of fractional quantum Hall physics, this mapping enabled precise experimental investigations of the dc-conductance scaling laws. The experiments reported in Refs. [69,71,72] provide stringent tests of the predicted universal critical behavior at low energies (temperature and dc voltage), even though the system is not strictly in the scaling limit because of the finite charging energy.

\section{Same equations but different solutions?}

At first sight, it is quite shocking that we invalidate the SB phase transition after the KF one was accurately confirmed; it obviously violates "the same equations have the same solutions" principle.

The key of this paradox is that the principle makes implicit assumptions on the equations' context. Everyone knows a given real-coefficient polynomial $p(x)$ may have roots or not depending whether the context is $x \in \mathbb{C}$ or $x \in \mathbb{R}$. The SB and KF systems can be described by the same effective action, but when one goes back to the underlying microscopic descriptions, different phenomenologies arise, providing different contexts for searching the solutions to the equations.

For a Josephson junction, there is a gap in the excitation spectrum of its electrodes. Consequently, after a slow enough $2 \pi$ phase slip, the junction is still in its ground state, and since the initial and final states of the junction are indiscernible, the junction's phase is compact. Superconductivity also yields a static phase coherence $\langle\cos \varphi\rangle \neq 0$, and an inductive response. Our work shows that this "superconducting context" is robust to connecting a resistor to the junction: The junction and the bath entangle, preserving finite coherences which forbids the phase transition. To put it more simply, in circuit engineering terms, the superconducting (ground-state) inductive response shunts the lowfrequency phase fluctuations arising from the series resistance; this makes the global system super-Ohmic, allowing the junction to preserve its superconducting character.

No such mechanism can take place in $\mathrm{KF}$ or $\mathrm{QPC}+R$ systems. In the case of an open $1 \mathrm{D}$ electonic channel with a barrier connected at both ends to reservoirs, a $2 \pi$ phase slip at the barrier (however slow) corresponds to a voltage pulse which, at $T=0$, can excite electrons and/or holes at arbitrarily low energy and which will be dissipated in the reservoirs. Thus, a phase slip takes this system to an orthogonal (distinguishable) state, such that the phase needs to be regarded as an extended phase. The Fermionic baths hence provide a subtle mechanism that is not contained in the equations of the effective model (where the Fermions no longer appear) and that allows breaking the discrete translational invariance of the phase in a way that totally differs from the partial decompatification mechanism we identify in JJs. Furthermore, in this system there is no possibility of a supercurrent in the ground state, and thus no static coherence $(\langle\cos \varphi\rangle=0)$. Connecting a resistor to the channel brings the system in the critical regime of the DQPT, with the expected localization effect described by Schmid and Bulgadaev. 
The above discussion in the main text suggests that the compactness of the phase-which we justify in the main text from the symmetry of the effective Josephson Hamiltonian-cannot be detached from the superconducting character of the Josephson junction and the existence of the inductive response.

\section{Superconducting-to-insulating transition in $1 \mathrm{D} \mathrm{JJ}$ arrays}

Another superconducting-to-insulating phase transition is predicted in 1D JJ arrays [73]. This latter transition was related to the disordered-induced transition (i.e., Anderson localization) predicted in fermionic 1D systems [74]. Recently, Kuzmin et al. investigated experimentally 1D JJ arrays and observed they remained good superconducting transmission lines well beyond the threshold line impedance predicted for their transition to the insulating state [75]. Given the similarities between that system and the one we consider, we believe it could be worth revisiting the predicted transition in $1 \mathrm{D} \mathrm{JJ}$ arrays taking into account what we understood on the sensitivity of the SB transition to the superconducting character of the underlying system.

[1] A. Schmid, Diffusion and Localization in a Dissipative Quantum System, Phys. Rev. Lett. 51, 1506 (1983).

[2] S. A. Bulgadaev, Phase Diagram of a Dissipative Quantum System, JETP Lett. 39, 315 (1984).

[3] F. Guinea, V. Hakim, and A. Muramatsu, Diffusion and Localization of a Particle in a Periodic Potential Coupled to a Dissipative Environment, Phys. Rev. Lett. 54, 263 (1985).

[4] C. Aslangul, N. Pottier, and D. Saint-James, Quantum Ohmic Dissipation: Particle on a One-Dimensional Periodic Lattice, Phys. Lett. 111A, 175 (1985).

[5] A. D. Zaikin and S. V. Panyukov, Dynamics of a Quantum Dissipative System: Duality between Coordinate and Quasimomentum Spaces, Phys. Lett. A 120, 306 (1987).

[6] G. Schön and A. D. Zaikin, Quantum Coherent Effects, Phase Transitions, and the Dissipative Dynamics of Ultra Small Tunnel Junctions, Phys. Rep. 198, 237 (1990).

[7] G.-L. Ingold and H. Grabert, Effect of Zero Point Phase Fluctuations on Josephson Tunneling, Phys. Rev. Lett. 83, 3721 (1999).

[8] C. P. Herrero and A. D. Zaikin, Superconductor-Insulator Quantum Phase Transition in a Single Josephson Junction, Phys. Rev. B 65, 104516 (2002).

[9] N. Kimura and T. Kato, Temperature Dependence of ZeroBias Resistances of a Single Resistance-Shunted Josephson Junction, Phys. Rev. B 69, 012504 (2004).

[10] H. Kohler, F. Guinea, and F. Sols, Quantum Electrodynamic Fluctuations of the Macroscopic Josephson Phase, Ann. Phys. (Amsterdam) 310, 127 (2004).

[11] P. Werner and M. Troyer, Efficient Simulation of Resistively Shunted Josephson Junctions, Phys. Rev. Lett. 95, 060201 (2005).
[12] S. L. Lukyanov and P. Werner, Resistively Shunted Josephson Junctions: Quantum Field Theory Predictions versus Monte Carlo Results, J. Stat. Mech. (2007) P06002.

[13] R. Yagi, S.-i. Kobayashi, and Y. Ootuka, Phase Diagram for Superconductor-Insulator Transition in Single Small Josephson Junctions with Shunt Resistor, J. Phys. Soc. Jpn. 66, 3722 (1997).

[14] J. S. Penttilä, Ü. Parts, P. J. Hakonen, M. A. Paalanen, and E. B. Sonin, Superconductor-Insulator Transition in a Single Josephson Junction, Phys. Rev. Lett. 82, 1004 (1999).

[15] L. S. Kuzmin, Yu. V. Nazarov, D. B. Haviland, P. Delsing, and T. Claeson, Coulomb Blockade and Incoherent Tunneling of Cooper Pairs in Ultrasmall Junctions Affected by Strong Quantum Fluctuations, Phys. Rev. Lett. 67, 1161 (1991).

[16] V. Bouchiat, D. Vion, P. Joyez, D. Esteve, and M. H. Devoret, Quantum Coherence with a Single Cooper Pair, Phys. Scr. T76, 165 (1998).

[17] Y. Nakamura, Yu. A. Pashkin, and J. S. Tsai, Coherent Control of Macroscopic Quantum States in a SingleCooper-Pair Box, Nature (London) 398, 786 (1999).

[18] J. Koch, T. M. Yu, J. Gambetta, A. A. Houck, D. I. Schuster, J. Majer, A. Blais, M. H. Devoret, S. M. Girvin, and R. J. Schoelkopf, Charge-Insensitive Qubit Design Derived from the Cooper Pair Box, Phys. Rev. A 76, 042319 (2007).

[19] J. A. Schreier, A. A. Houck, J. Koch, D. I. Schuster, B. R. Johnson, J. M. Chow, J. M. Gambetta, J. Majer, L. Frunzio, M. H. Devoret, S. M. Girvin, and R. J. Schoelkopf, Suppressing Charge Noise Decoherence in Superconducting Charge Qubits, Phys. Rev. B 77, 180502 (2008).

[20] F. Nguyen, N. Boulant, G. Ithier, P. Bertet, H. Pothier, D. Vion, and D. Esteve, Current to Frequency Conversion in a Josephson Circuit, Phys. Rev. Lett. 99, 187005 (2007).

[21] H. Grabert, U. Weiss, and P. Talkner, Quantum Theory of the Damped Harmonic Oscillator, Z. Phys. B 55, 87 (1984).

[22] U. Vool and M. Devoret, Introduction to Quantum Electromagnetic Circuits, Int. J. Circuit Theory Appl. 45, 897 (2017).

[23] P. Joyez, Self-Consistent Dynamics of a Josephson Junction in the Presence of an Arbitrary Environment, Phys. Rev. Lett. 110, 217003 (2013).

[24] S. E. Nigg, H. Paik, B. Vlastakis, G. Kirchmair, S. Shankar, L. Frunzio, M. H. Devoret, R. J. Schoelkopf, and S. M. Girvin, Black-Box Superconducting Circuit Quantization, Phys. Rev. Lett. 108, 240502 (2012).

[25] J. Koch, V. Manucharyan, M. H. Devoret, and L. I. Glazman, Charging Effects in the Inductively Shunted Josephson Junction, Phys. Rev. Lett. 103, 217004 (2009).

[26] For devices made out of a BCS superconductor (with a gap $\Delta$ ) and which conduct in the normal state, at frequencies $f \ll \Delta / h$ and temperatures $T \ll \Delta / k_{B}$, the linear response is dominated by an inductive behavior. For a Josephson junction, the effective inductance $L_{J}^{\text {eff }}$ is at least $\hbar^{2} / 4 e^{2} E_{J}$ and can be enlarged due to environment-induced phase fluctuations [23]. An insulating behavior would correspond to a divergence of this inductance. See also Appendix F.

[27] B. Huard, H. Pothier, D. Esteve, and K. E. Nagaev, Electron Heating in Metallic Resistors at Sub-Kelvin Temperature, Phys. Rev. B 76, 165426 (2007).

[28] M. Vojta, Quantum Phase Transitions, Rep. Prog. Phys. 66, 2069 (2003). 
[29] A. Grimm, F. Blanchet, R. Albert, J. Leppäkangas, S. Jebari, D. Hazra, F. Gustavo, J.-L. Thomassin, E. Dupont-Ferrier, F. Portier, and M. Hofheinz, Bright On-Demand Source of Antibunched Microwave Photons Based on Inelastic Cooper Pair Tunneling, Phys. Rev. X 9, 021016 (2019).

[30] J. S. Penttilä, P. J. Hakonen, E. B. Sonin, and M. A. Paalanen, Experiments on Dissipative Dynamics of Single Josephson Junctions, J. Low Temp. Phys. 125, 89 (2001).

[31] A. O. Caldeira and A. J. Leggett, Quantum Tunnelling in a Dissipative System, Ann. Phys. (N.Y.) 149, 374 (1983).

[32] Note that in standard CPB circuits where the junction is isolated from the dissipative environment by a gate capacitor not allowing a dc charge flow, this translational invariance of the charge is suppressed, and the quasicharge is a relevant parameter.

[33] K. Mullen, D. Loss, and H. T. C. Stoof, Resonant Phenomena in Compact and Extended Systems, Phys. Rev. B 47, 2689 (1993).

[34] D. Loss and K. Mullen, Effect of Dissipation on Phase Periodicity and the Quantum Dynamics of Josephson Junctions, Phys. Rev. A 43, 2129 (1991).

[35] S. M. Apenko, Environment-Induced Decompactification of Phase in Josephson Junctions, Phys. Lett. A 142, 277 (1989).

[36] M. P. A. Fisher and W. Zwerger, Quantum Brownian Motion in a Periodic Potential, Phys. Rev. B 32, 6190 (1985).

[37] H. Spohn, Ground State(s) of the Spin-Boson Hamiltonian, Commun. Math. Phys. 123, 277 (1989).

[38] K. Le Hur, Entanglement Entropy, Decoherence, and Quantum Phase Transitions of a Dissipative Two-Level System, Ann. Phys. (Amsterdam) 323, 2208 (2008).

[39] S. Bera, S. Florens, H. U. Baranger, N. Roch, A. Nazir, and A. W. Chin, Stabilizing Spin Coherence through Environmental Entanglement in Strongly Dissipative Quantum Systems, Phys. Rev. B 89, 121108 (2014).

[40] S. Bera, A. Nazir, A. W. Chin, H. U. Baranger, and S. Florens, Generalized Multipolaron Expansion for the SpinBoson Model: Environmental Entanglement and the Biased Two-State System, Phys. Rev. B 90, 075110 (2014).

[41] H. Grabert unpublished.

[42] U. Weiss, Quantum Dissipative Systems, 4th ed. (World Scientific, Singapore, 2012), https://doi.org/10.1142/8334.

[43] A. J. Kerman, Flux-Charge Duality and Topological Quantum Phase Fluctuations in Quasi-One-Dimensional Superconductors, New J. Phys. 15, 105017 (2013).

[44] J. E. Mooij and Yu. V. Nazarov, Superconducting Nanowires as Quantum Phase-Slip Junctions, Nat. Phys. 2, 169 (2006).

[45] J. Ulrich and F. Hassler, Dual Approach to Circuit Quantization Using Loop Charges, Phys. Rev. B 94, 094505 (2016).

[46] P. Joyez, D. Vion, M. Götz, M. H. Devoret, and D. Esteve, The Josephson Effect in Nanoscale Tunnel Junctions, J. Supercond. 12, 757 (1999).

[47] A. Steinbach, P. Joyez, A. Cottet, D. Esteve, M. H. Devoret, M. E. Huber, and J. M. Martinis, Direct Measurement of the Josephson Supercurrent in an Ultrasmall Josephson Junction, Phys. Rev. Lett. 87, 137003 (2001).

[48] D. Vion, M. Götz, P. Joyez, D. Esteve, and M. H. Devoret, Thermal Activation above a Dissipation Barrier: Switching of a Small Josephson Junction, Phys. Rev. Lett. 77, 3435 (1996).
[49] D. M. Pozar, Microwave Engineering, 4th ed. (John Wiley \& Sons, Hoboken, NJ, 2011).

[50] C. Rolland, A. Peugeot, S. Dambach, M. Westig, B. Kubala, Y. Mukharsky, C. Altimiras, H. le Sueur, P. Joyez, D. Vion, P. Roche, D. Esteve, J. Ankerhold, and F. Portier, Antibunched Photons Emitted by a dc-Biased Josephson Junction, Phys. Rev. Lett. 122, 186804 (2019).

[51] S. Léger, J. Puertas-Martínez, K. Bharadwaj, R. Dassonneville, J. Delaforce, F. Foroughi, V. Milchakov, L. Planat, O. Buisson, C. Naud, W. Hasch-Guichard, S. Florens, I. Snyman, and N. Roch, Observation of Quantum ManyBody Effects due to Zero Point Fluctuations in Superconducting Circuits, Nat. Commun. 10, 5259 (2019).

[52] I. Safi and P. Joyez, Time-Dependent Theory of Nonlinear Response and Current Fluctuations, Phys. Rev. B 84, 205129 (2011).

[53] G.-L. Ingold and Yu. V. Nazarov, in Single Charge Tunneling, edited by $\mathrm{H}$. Grabert and M. H. Devoret, NATO ASI Series B Vol. 294 (Plenum Press, New York, 1992), pp. 21-107.

[54] M. Hofheinz, F. Portier, Q. Baudouin, P. Joyez, D. Vion, P. Bertet, P. Roche, and D. Esteve, Bright Side of the Coulomb Blockade, Phys. Rev. Lett. 106, 217005 (2011).

[55] D. V. Averin, A. B. Zorin, and K. K. Likharev, Bloch Oscillations in Small Josephson Junctions, Sov. Phys. JETP 61, 407 (1985).

[56] W. Zwerger, A. T. Dorsey, and M. P. A. Fisher, Effects of the Phase Periodicity on the Quantum Dynamics of a Resistively Shunted Josephson Junction, Phys. Rev. B 34, 6518 (1986).

[57] V. E. Manucharyan, J. Koch, L. I. Glazman, and M. H. Devoret, Fluxonium: Single Cooper-Pair Circuit Free of Charge Offsets, Science 326, 113 (2009).

[58] G. Burkard, R. H. Koch, and D. P. DiVincenzo, Multilevel Quantum Description of Decoherence in Superconducting Qubits, Phys. Rev. B 69, 064503 (2004).

[59] G. Burkard, Circuit Theory for Decoherence in Superconducting Charge Qubits, Phys. Rev. B 71, 144511 (2005).

[60] V. Ambegaokar and A. Baratof, Tunneling between Superconductors, Phys. Rev. Lett. 10, 486 (1963).

[61] V. Ambegaokar, U. Eckern, and G. Schön, Quantum Dynamics of Tunneling between Superconductors, Phys. Rev. Lett. 48, 1745 (1982).

[62] J. M. Martinis, M. Ansmann, and J. Aumentado, Energy Decay in Superconducting Josephson-Junction Qubits from Nonequilibrium Quasiparticle Excitations, Phys. Rev. Lett. 103, 097002 (2009).

[63] M. H. Ansari, F. K. Wilhelm, U. Sinha, and A. Sinha, The Effect of Environmental Coupling on Tunneling of Quasiparticles in Josephson Junctions, Supercond. Sci. Technol. 26, 125013 (2013).

[64] E. Dalla Torre and E. Sela, Viewpoint: Circuit Simulates One-Dimensional Quantum System, Physics 11, 94 (2018).

[65] C. L. Kane and M. P. A. Fisher, Transport in a One-Channel Luttinger Liquid, Phys. Rev. Lett. 68, 1220 (1992).

[66] X. G. Wen, Electrodynamical Properties of Gapless Edge Excitations in the Fractional Quantum Hall States, Phys. Rev. Lett. 64, 2206 (1990).

[67] C. L. Kane and M.P. A. Fisher, Transmission through Barriers and Resonant Tunneling in an Interacting OneDimensional Electron Gas, Phys. Rev. B 46, 15233 (1992). 
[68] P. Fendley, A. W. W. Ludwig, and H. Saleur, Exact Conductance through Point Contacts in the $\nu=1 / 3$ Fractional Quantum Hall Effect, Phys. Rev. Lett. 74, 3005 (1995).

[69] A. Anthore, Z. Iftikhar, E. Boulat, F. D. Parmentier, A. Cavanna, A. Ouerghi, U. Gennser, and F. Pierre, Circuit Quantum Simulation of a Tomonaga-Luttinger Liquid with an Impurity, Phys. Rev. X 8, 031075 (2018).

[70] I. Safi and H. Saleur, One-Channel Conductor in an Ohmic Environment: Mapping to a Tomonaga-Luttinger Liquid and Full Counting Statistics, Phys. Rev. Lett. 93, 126602 (2004).

[71] S. Jezouin, M. Albert, F. D. Parmentier, A. Anthore, U. Gennser, A. Cavanna, I. Safi, and F. Pierre, TomonagaLuttinger Physics in Electronic Quantum Circuits, Nat. Commun. 4, 1802 (2013).
[72] F. D. Parmentier, A. Anthore, S. Jezouin, H. le Sueur, U. Gennser, A. Cavanna, D. Mailly, and F. Pierre, Strong Back-Action of a Linear Circuit on a Single Electronic Quantum Channel, Nat. Phys. 7, 935 (2011).

[73] R. M. Bradley and S. Doniach, Quantum Fluctuations in Chains of Josephson Junctions, Phys. Rev. B 30, 1138 (1984).

[74] T. Giamarchi and H. J. Schulz, Anderson Localization and Interactions in One-Dimensional Metals, Phys. Rev. B 37, 325 (1988).

[75] R. Kuzmin, R. Mencia, N. Grabon, N. Mehta, Y.-H. Lin, and V.E. Manucharyan, Quantum Electrodynamics of a Superconductor-Insulator Phase Transition, Nat. Phys. 15, 930 (2019). 